\title{
Mixed Problems for Singular and Degenerate Hyperbolic Equations
}

By

\section{Akisato KUBO*}

We start with a simple example. Let us consider the mixed problem for a weakly hyperbolic equation of third order:

$$
A=\left(D_{t}-a t^{1 / 2} D_{x}\right)\left(D_{t}^{2}-t D_{x}^{2}\right)+\text { any smooth lower order terms, }
$$

where $(t, x) \in(0, T) \times \mathbb{R}_{+}, D_{t}=-i(\partial / \partial t), D_{x}=-i(\partial / \partial x)$ and $a$ is a positive constant $\neq 1$. Since the principal symbol of $A$ is of the form:

$$
A_{0}(\tau, \xi)=\left(\tau-a t^{1 / 2} \xi\right)\left(\tau^{2}-t \xi^{2}\right),
$$

$A_{0}(\tau, \xi)=0$ as the equation of $\xi$ has the roots $\xi_{+}$with positive imaginary part and $\xi_{-}^{1}, \xi_{-}^{2}$ each with negative imaginary part for $\operatorname{Im} \tau<0$. For the mixed problem considered to be well posed the number of boundary operators to be given should be equal to that of roots $\xi$ of $A_{0}(\tau, \xi)=0$ with $\operatorname{Im} \xi>0$. Therefore to the above example we may give a boundary condition $\left.u\right|_{x=0}=g(t)$, for example. Then in our paper it will be shown that there exists the unique solution $u \in H_{\infty}\left((0, T) \times \mathbb{R}_{+}\right)$of the following problem. Since we consider $t D_{t}$ and $t^{3 / 2} D_{x}$ instead of $D_{t}$ and $D_{x}$ respectively, we write $A[u]$ as follows.

$$
\left\{\begin{aligned}
& A[u]=t^{-3}\left\{\left(t D_{t}-a t^{3 / 2} D_{x}\right)\left(\left(t D_{t}\right)^{2}-t^{3} D_{x}^{2}\right)+\text { lower order terms }\right\} \\
&=f(t, x) \in H_{\infty}\left((0, T) \times \mathbb{R}_{+}\right), \\
&\left.u\right|_{x=0}=g(t) \in H_{\infty}(0, T), \\
& \text { all } t \text {-derivatives of } f \text { and } g \text { are zero at } t=0 .
\end{aligned}\right.
$$

Now we consider the problem in the more general situation.

Let $P$ and $B_{k}\left(k=1, \ldots, m_{+}\right)$be given by

Communicated by S. Matsuura, March 8, 1986.

* Department of Mathematics, Science and Engineering, Waseda University, Ohkubo, Shinjuku, Tokyo 160, Japan.

Current address: School of Hygiene, Fujita-Gakuen Health University, Toyoake, Aichi 47011, Japan. 


$$
\begin{aligned}
P & =P\left(t, x, y ; t D_{t}, D_{x}, D_{y}\right) \\
& =\sum_{s=0}^{m} \sum_{i+j+|\nu|=m-s} t^{\kappa(j+|\nu|)} a_{i j \nu}(t, x, y)\left(t D_{t}\right)^{i} D_{x}^{j} D_{y}^{\nu}
\end{aligned}
$$

with $a_{m 00} \neq 0, a_{0 m 0}=1$, a real number $\kappa>0$ and

$$
\begin{aligned}
B_{k} & =B_{k}\left(t, y ; t D_{t}, D_{x}, D_{y}\right) \\
& =\sum_{s=0}^{r_{k}} \sum_{i+j+|\nu|=r_{k}-s} t^{k(j+|\nu|)} b_{i j \nu}^{k}(t, y)\left(t D_{t}\right)^{i} D_{x}^{j} D_{y}^{\nu}
\end{aligned}
$$

with $b_{0, r_{k}, 0}^{k}=1$ for $0 \leqq r_{k} \leqq m-1, r_{i} \neq r_{j}(i \neq j)$.

We assume all the coefficients of $\left\{P, B_{k}\right\}$ are smooth in $\boldsymbol{R}_{+} \times \boldsymbol{R}^{n}$. Furthermore we impose the following assumption (A) on $\left\{P, B_{k}\right\}$ :

(A) $-\mathrm{i} \quad$ For the set $K=\left\{(t, x, y) \in \boldsymbol{R}_{+} \times \boldsymbol{R}^{n} ;|(t, x, y)| \leqq T_{0}\right\}$ all the coefficients of $\left\{P, B_{k}\right\}$ are constant in $\boldsymbol{R}_{+}^{n+1} \backslash K$.

(A)-ii $\quad P$ is hyperbolic with respect to $t$, that is, the principal symbol of $P$ is of the form:

$$
P_{m}(t, x, y ; \tau, \xi, \eta)=\prod_{j=1}^{m}\left(\tau-t^{\kappa} \Lambda_{j}(t, x, y ; \xi, \eta)\right),(\xi, \eta) \in \boldsymbol{R} \times \boldsymbol{R}^{n-1}
$$

where $\Lambda_{j}(t, x, y ; \xi, \eta)$ are real and distinct. Hence when $(t, x, y) \in \boldsymbol{R}_{+}^{n+1}$, $\operatorname{Im} \tau<0$ and $\eta \in \mathbb{R}^{n-1}, P_{m}=0$ has only non-real roots with respect to $\xi$. Let $m_{+}$be the number of roots with positive imaginary part. We denote such roots by $\left\{\xi_{j 0}^{+}\left(t, x, y ; t^{-\kappa} \tau, \eta\right)\right\}_{1 \leqq j \leqq m_{+}} \cdot$

(A) -iii $\quad\left\{P, B_{k}\right\}$ satisfy the uniform Lopatinski's condition on $x=0$, that is, for the Lopatinski's determinant

$$
R_{0}(t, y ; \theta, \eta)=\operatorname{det}\left(\frac{1}{2 \pi i} \oint \frac{\tilde{B}_{k 0}(t, y ; \theta, \xi, \eta) \xi^{j-1}}{\prod_{i=1}^{m_{+}}\left(\xi-\xi_{i 0}^{+}(t, 0, y ; \theta, \eta)\right)} d \xi\right)_{j, k=1, \ldots, m_{+}}
$$

$R_{0}(t, y ; \theta, \eta) \neq 0$ holds for $(t, y) \in[0, \infty) \times \boldsymbol{R}^{n-1}, \operatorname{Im} \theta \leqq 0, \eta \in \boldsymbol{R}^{n-1}$ and $(\theta, \eta) \neq(0,0)$, where $\tilde{B}_{k 0}\left(t, y ; D_{t}, D_{y}, D_{x}\right)=\sum_{i+j+|\nu|=r_{k}} b_{i j \nu}^{k}(t, y) D_{t}^{i} D_{x}^{j} D_{y^{*}}^{\nu}$

In case of the above example, $P\left(t, x ; D_{t}, D_{x}\right)=t^{3} A[u]=\left(t D_{t}\right)^{3}-$ $a\left(t D_{t}\right)^{2}\left(t^{3 / 2} D_{x}\right)-\left(t D_{t}\right)\left(t^{3 / 2} D_{x}\right)^{2}+a\left(t^{3 / 2} D_{x}\right)^{3}+$ lower order terms and in (0.4) actually we have $m=3, P_{3}(t, x ; \tau, \xi)=\left(t^{3} A\right)_{0}(\tau, \xi), \kappa=3 / 2, \Lambda_{1}=a \xi, \Lambda_{2}=\xi$, $\Lambda_{3}=-\xi$.

Now we consider a flat mixed problem for $\left\{P, B_{k}\right\}$ satisfying the assumption (A). 


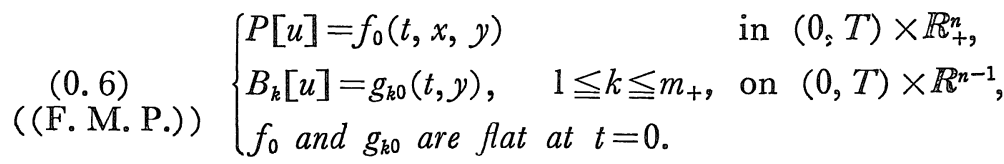

Recently Sakamoto [10] considered the following flat mixed problem for a degenerate hyperbolic equation of order $m$ :

$$
\mathscr{A}\left(t, x ; D_{t}, D_{x}\right)=\sum_{j=0}^{m} \sum_{i+|\nu|=m-j} a_{j \nu}(t, x) t^{\gamma_{1}+\ldots+r_{|\nu|}}\left(t D_{t}\right)^{i} D_{x}^{\nu}
$$

with

$$
\begin{gathered}
\gamma_{0}=0 \leqq \gamma_{1}=\ldots=\gamma_{m_{1}}<\gamma_{m_{1}+1}=\ldots=\gamma_{m_{1}+m_{2}}<\ldots<\gamma_{m_{1}+\ldots+m_{l-1}+1} \\
=\ldots=\gamma_{m_{1}+\ldots+m_{l}}\left(m_{1}+\ldots+m_{l}=m\right), x=\left(x^{\prime}, x_{n}\right) \in \mathbb{R}^{n-1} \times \mathbb{R}_{+}
\end{gathered}
$$

and $t \in(0, T)$, with the boundary operators on $x_{n}=0$ :

$$
\mathscr{B}_{k}\left(t, x^{\prime} ; D_{t}, D_{x}\right)=\sum_{j=0}^{r_{k}} \sum_{i+|\nu|=r_{k}-j} b_{j \nu}^{k}\left(t, x^{\prime}\right) t^{\hat{r}_{0}+\ldots+\hat{r}_{|\nu|}}\left(t D_{t}\right)^{i} D_{x}^{\nu}
$$

with $\hat{\gamma}_{j}=\left(\gamma_{j}+\gamma_{j+1}\right) / 2,1 \leqq k \leqq m / 2$ and $m$ is even. $\mathscr{A}$ has more degeneracy than our operator $P$ at $t=0$ and $\left\{\mathscr{B}_{j}\right\}$ are boundary operators associated with $\mathscr{A}$. Then she gave a sufficient condition for the problem to be $H_{\infty}$-well posed and then showed that in case $\gamma_{1}>0$ the problem is $C^{\infty}$-well posed. However her result in [10] is not a full extension of $[8 ; 9]$ in such a sense that on the equations some stronger conditions are assumed in [10] than in [8;9].

The aim of this paper is to obtain a complete extension of Sakamoto's result in $[8 ; 9]$ without assuming $m$ to be even. We shall consider such mixed problem with flat data at $t=0$ in $\S 1 \sim \S 4$ and with data which are not always flat at $t=0$ in Appendix。O Our main result is as follows.

Theorem 0.1. For given flat data: $f_{0} \in H_{\infty}\left((0, T) \times \mathbb{R}_{+}^{n}\right)$ and $g_{j 0} \in$ $H_{\infty}\left((0, T) \times \mathbb{R}^{n-1}\right)\left(1 \leqq j \leqq m_{+}\right)$there exists the unique solution $u$ of (F.M.P.) in $H_{\infty}\left((0, T) \times \mathbb{R}_{+}^{n}\right)$. For any integer $s \geqq 0$ there exist a constant $G$, integers $N$ and $N_{j}\left(j=1, \ldots, m_{+}\right)$such that the estimate

$$
\begin{aligned}
\|u\|_{H_{m+s-1}\left((0, T) \times R_{+}^{n}\right)}^{2} & \leqq C\left\{\left\|f_{0}\right\|_{H_{s+N}\left((0, T) \times R_{+}^{n}\right)}^{2}\right. \\
& +\sum_{j=1}^{m_{+}}\left\|g_{j 0}\right\|_{H_{m-1-r_{j}+s+N_{j}}}^{2}\left((0, T) \times R^{n-1}\right)
\end{aligned}
$$

holds. 
Remark 1. $C, N$ and $N_{j}\left(1 \leqq j \leqq m_{+}\right)$depend on $\left\|D_{t}^{k} D_{x}^{l} D_{y}^{\mu} a_{i j \nu}(t, x, y)\right\|_{L^{\infty}}$ for $k+l+|\mu| \leqq \max (2, m-1, s), i+j+|\nu|=m$, $\left\|D_{t}^{k} D_{x}^{l} D_{y}^{\mu} a_{i j \nu}(t, x, y)\right\|_{L^{\infty}}$ for $k+l+|\mu| \leqq s, i+j+|\nu| \leqq m-1$ and $\left\|D_{t}^{l} D_{y}^{\mu} b_{i j \nu}^{k}(t, y)\right\|_{L^{\infty}}$ for $l+|\mu| \leqq s+m-1-r_{k}, i+j+|\nu| \leqq r_{k}, 1 \leqq k \leqq m_{+}$.

This theorem can be applied to the following type of operators.

\section{Example 1. (Toricomi's operator)}

$$
D_{t}^{2}-t\left(D_{x}^{2}+D_{y}^{2}\right)=t^{-2}\left\{\left(t D_{t}\right)^{2}-t^{3}\left(D_{x}^{2}+D_{y}^{2}\right)+\text { lower order terms }\right\}
$$

in $(t, x, y) \in(0, T) \times \mathbb{R}_{+} \times \mathbb{R}$ with the boundary operator on $x=0$ :

$$
\begin{aligned}
& a D_{t}+t^{1 / 2}\left(-D_{x}+b D_{y}\right)=t^{-1}\left\{a t D_{t}+t^{3 / 2}\left(-D_{x}+b D_{y}\right)\right\} \\
& \quad\left(a, b \in \mathbb{R}^{1}, a>|b|\right) .
\end{aligned}
$$

\section{2. (Euler-Poisson-Darboux operator)}

$$
D_{t}^{2}-\sum_{i=1}^{n} D_{x_{i}}^{2}+t^{-1} D_{t}=t^{-2}\left\{\left(t D_{t}\right)^{2}-\sum_{i=1}^{n}\left(t D_{x_{i}}\right)^{2}+t D_{t}+\text { lower order terms }\right\}
$$

in $\left(t, x^{\prime}, x_{n}\right) \in(0, T) \times \boldsymbol{R}^{n-1} \times \boldsymbol{R}_{+}$with the boundary operator on $x_{n}=0$ :

$$
\begin{gathered}
a D_{t}+\left(-D_{x_{n}}+b D_{x^{\prime}}\right)=t^{-1}\left\{a t D_{t}+t\left(-D_{x_{n}}+b D_{x^{\prime}}\right)\right\} \\
\left(a, b \in \mathbb{R}^{1}, a>|b|\right) .
\end{gathered}
$$

Our method is summerized as follows.

By the substitution $s=t^{\kappa}, P$ is reduced to an operator:

$$
\begin{aligned}
P & =s^{m} \tilde{P}\left(s, x, y: D_{s}, D_{x}, D_{y}\right)=s^{m}\left\{P_{m}\left(s^{1 / \kappa}, x, y ; \kappa D_{s}, D_{x}, D_{y}\right)\right. \\
& \left.+\sum_{l=1}^{m} \sum_{i+j+|\nu| \leqq m-l} a_{i j \nu}^{\prime}\left(s^{1 / \kappa}, x, y\right) s^{-l} D_{s}^{i} D_{x}^{j} D_{y}^{\nu}\right\}
\end{aligned}
$$

where $a_{i j \nu}^{\prime}(t, x, y)$ are suitable smooth functions and $\tilde{P}$ is regularly hyperbolic, however the coefficients of $\tilde{P}$ are not smooth. In the same procedure, we define $\tilde{B}_{k}\left(s, y ; D_{s}, D_{x}, D_{y}\right)$. Our method is based heavily on the energy estimate for $\left\{\tilde{P}, \tilde{B}_{k}\right\}$. Following [8;9], the desired estimate is derived from estimates for some singular integral operators with a parameter $\gamma>0$. In [8;9] for this purpose sufficient smoothness was needed for their corresponding symbols. However in our case the symbols are not smooth at $s=0$. To overcome this difficulty we must investigate the properties of the operator $H(t, y ; \gamma$, $\left.D_{t}, D_{y}\right)$ with the symbol $h(t, y ; \gamma, \tau, \eta)$ satisfying $\left(g(t) D_{t}\right)^{j} D_{y}^{\nu} h \in \mathscr{B}_{\alpha, k}\left(\mathbb{R}^{n}\right)$ 
$(j+|\nu| \leqq 1)$ where $\mathscr{B}_{\alpha, k}$ is a non-regular symbol class introduced in Sakamoto [7] and $g(t) \in \mathscr{B}^{\infty}(\mathbb{R})$ and is equal to $t$ in a neighborhood of the origin. For such singular integral operators we obtain some basic estimates, for example,

$$
\begin{aligned}
\left\|e^{-\gamma t}\left(H_{1} \circ H_{2}-H_{1} H_{2}\right) \Lambda g(t) u\right\|_{L^{2}\left(R^{n}\right)} \leqq & C\left\{(1+\gamma)\left\|g(t) e^{-\gamma t} u\right\|_{L^{2}\left(R^{n}\right)}\right. \\
& \left.+\left\|e^{-\gamma t} u\right\|_{L^{2}\left(R^{n}\right)}\right\}
\end{aligned}
$$

where $H_{1}$ and $H_{2}$ are such operators. By making use of these estimates we can derive the desired estimate.

This paper is organized as follows. In the first section the existence and uniqueness theorem is proved from an energy inequality by an approximation method. In Section 2 we shall investigate the property of the singular integral operators with non-regular symbols and in Section 3 we derive some basic estimates for them. The final section is devoted to the proof of the energy inequality for (F. M. $\mathrm{P}_{0}$ ) used in Section 1. In Appendix we consider a degenerate hyperbolic mixed problem with data which are not necessarily flat at $t=0$.

The author would like to express his sincere gratitude to Professor T. Kakita for suggesting the problem and helpful discussions. Also his thanks are due to Professor R. Sakamoto and Professor H. Uryu for their valuable advices.

\section{§1. Existence and Uniqueness}

We introduce a new independent variable

$$
s=t^{\kappa}
$$

(see [4]). Then we observe that

$$
\begin{aligned}
& t(\partial / \partial t)=\kappa t^{\kappa} \partial / \partial s, \\
& \left(\tau=\kappa t^{\kappa} \tau^{\prime}\right)
\end{aligned}
$$

where $\tau^{\prime}$ is a dual variable of $s$. We denote $\tilde{a}(s, x, y)=a(t, x, y)$. Then we have

$$
\begin{aligned}
P(t, x, y ; \tau, \xi, \eta) & =\sum_{l=0}^{m} \sum_{i+j+|\nu|=m-l} \tilde{a}_{i j \nu}(s, x, y) s^{j+\mid \nu i}\left(\kappa s \tau^{\prime}\right)^{i} \xi^{j} \eta^{\nu} \\
& =s^{m}\left(\sum_{l=0}^{m} \sum_{i+j+|\nu|=m-l} \tilde{a}_{i j \nu}(s, x, y) s^{-l}\left(\kappa \tau^{\prime}\right)^{i} \xi^{j} \eta^{\nu}\right) .
\end{aligned}
$$

Thus, there exist smooth functions $a_{i j \nu}^{\prime}(t, x, y)$ satisfying (A) $-\mathrm{i}$ such 
that

$$
\begin{gathered}
P\left(t, x, y ; D_{t}, D_{x}, D_{y}\right)=s^{m}\left\{\sum_{i+j+|\nu|=m} \tilde{a}_{i j \nu}(s, x, y)\left(\kappa D_{s}\right)^{i} D_{x}^{j} D_{y}^{\nu}\right. \\
\left.+\sum_{l=1}^{m} \sum_{i+j+|\nu|=m-l} \tilde{a}_{i j \nu}^{\prime}(s, x, y) s^{-l} D_{s}^{i} D_{x}^{j} D_{y}^{\nu}\right\} .
\end{gathered}
$$

Then we introduce a smooth function $b(s)$ such that $b(s)=s$ in $\left\{|s| \leqq T_{0}{ }^{\kappa}+1\right\}$ and constant in $\left\{|s| \geqq T_{0}{ }^{\kappa}+2\right\}$ where $T_{0}$ is appeared in (A) -i. Put, for $(s, x, y) \in \boldsymbol{R}_{+} \times \boldsymbol{R}_{+}^{n}$,

$$
\begin{aligned}
\tilde{P}\left(s, x, y ; D_{s}, D_{x}, D_{y}\right) & =\sum_{i+j+|\nu|=m} \tilde{a}_{i j \nu}(s, x, y)\left(\kappa D_{s}\right)^{i} D_{x}^{j} D_{y}^{\nu} \\
& +\sum_{l=1}^{m} \sum_{i+j+|\nu|=m-l} \tilde{a}_{i j \nu}^{\prime}(s, x, y) b(s)^{-l} D_{s}^{i} D_{x}^{j} D_{y}^{\nu} .
\end{aligned}
$$

In the same procedure we define

$$
\begin{aligned}
\tilde{B}_{k}\left(s, y ; D_{s}, D_{x}, D_{y}\right) & =\sum_{i+j+|\nu|=r_{j}} \tilde{b}_{i j \nu}^{k}(s, y)\left(\kappa D_{s}\right)^{i} D_{x}^{j} D_{y}^{\nu} \\
& +\sum_{l=1}^{r_{j}} \sum_{i+j+|\nu|=r_{j}-l} \tilde{b}_{i j \nu}^{k^{\prime}}(s, y) b(s)^{-l} D_{s}^{i} D_{x}^{j} D_{y .}^{\nu}
\end{aligned}
$$

Then (F. M. P.) can be reduced to the problem: for $T_{1}=T^{\kappa}$,

$$
\left\{\begin{array}{l}
\tilde{P}[u]=b(s)^{-m} \tilde{f}_{0}(s, x, y)=f_{1}(s, x, y) \text { in }\left(0, T_{1}\right) \times \boldsymbol{R}_{+}^{n} \\
\tilde{B}_{k}[u]=b(s)^{-r_{k}} \tilde{g}_{k 0}(s, y)=g_{k 1}(s, y) \text { on }\left(0, T_{1}\right) \times \boldsymbol{R}^{n-1} .
\end{array}\right.
$$

Now we prove the existence of solution of (1.2) by the compactness method as follows. Let $s_{\varepsilon}=\left(s^{2}+\varepsilon\right)^{1 / 2}$ for a non-negative parameter $\varepsilon \geqq 0$. We write $a_{\varepsilon}(s, x, y)=a\left(s_{\varepsilon}, x, y\right)$. We define $\left\{\tilde{f}_{1}, \tilde{g}_{k 1}\right\}$ as extensions of $\left\{f_{1}, g_{k 1}\right\}$ such that $\left\{\tilde{f}_{1}, \tilde{g}_{k 1}\right\}$ have compact support in $\{s \geqq 0\}$ as function of $s$ and satisfy for any fixed $r \in \boldsymbol{N}$,

$$
\left\{\begin{array}{l}
\left\|\tilde{f}_{1}\right\|_{H_{\boldsymbol{r}}\left(\boldsymbol{R}_{+}^{n+1}\right)} \leqq C\left\|f_{1}\right\|_{H_{\boldsymbol{r}}\left(\left(0, T_{1}\right) \times R_{+}^{n}\right)} \\
\left\|\tilde{g}_{k}\right\|_{H_{\boldsymbol{r}}\left(\boldsymbol{R}^{n}\right)} \leqq C\left\|g_{k 1}\right\|_{H_{\boldsymbol{r}}\left(\left(0, T_{1}\right) \times R^{n-1}\right)}
\end{array}\right.
$$

We consider the following hyperbolic boundary problem:

$$
\left\{\begin{array}{lll}
\tilde{P}_{\varepsilon}[u]=\tilde{f}_{1}(s, x, y) & \text { in } & \boldsymbol{R} \times \boldsymbol{R}_{+}^{n} \\
\tilde{B}_{k \varepsilon}[u]=\tilde{g}_{k 1}(s, y) & \text { on } & \boldsymbol{R} \times \boldsymbol{R}^{n-1}
\end{array}\right.
$$

where $\tilde{P}_{\varepsilon}\left(\tilde{B}_{k \varepsilon}\right)$ is the operator defined by replacing $\tilde{a}_{i j \nu}, b(s)^{-l} \tilde{a}_{i j \nu}^{\prime}$ $\left(\tilde{b}_{i j \nu}^{k}, b(s)^{-l} \tilde{b}_{i j \nu}^{k^{\prime}}\right)$ by $\tilde{a}_{i j \nu, \varepsilon}, b_{\varepsilon}(s)^{-l} \tilde{a}_{i j \nu, \varepsilon}^{\prime}\left(\tilde{b}_{i j \nu, \varepsilon}^{k}, b_{\varepsilon}(s)^{-l} \tilde{b}_{i j \nu, \varepsilon}^{k^{\prime}}\right)$ in $\tilde{P}\left(\tilde{B}_{k}\right)$ respectively. We easily see that the compatibility between the data in (1.4) holds and that the uniform Lopatinski's condition for $\left\{\tilde{P}_{\varepsilon}, \tilde{B}_{k \varepsilon}\right\}$ holds from (A)-iii, i. e., for Lopatinski's determinant 


$$
R_{\varepsilon}\left(s, y ; \kappa \tau^{\prime}, \eta\right)=\operatorname{det}\left(\frac{1}{2 \pi i} \oint \frac{\tilde{B}_{k 0, \varepsilon}\left(s, y ; \kappa \tau^{\prime}, \xi, \eta\right) \xi^{j-1}}{\prod_{i=1}^{m_{+}}\left(\xi-\xi_{i 0}^{+}\left(s_{\varepsilon,} 0, y ; \kappa \tau^{\prime}, \eta\right)\right)} d \xi\right)_{j, k=1, \ldots, m_{+}}
$$

$\operatorname{Inf}_{\varepsilon>0}\left|R_{\varepsilon}\right| \neq 0$ holds for $\operatorname{Im} \tau^{\prime} \leqq 0, \eta \in \mathbb{R}^{n-1}, \quad\left(\tau^{\prime}, \eta\right) \neq(0,0)$ and $(s, y) \in \mathbb{R}^{n}$. Therefore the existence of unique solution $u_{\varepsilon} \in H_{m-1+r}\left(\left(0, T_{0}^{\kappa}\right) \times \mathbb{R}_{+}^{n}\right)$ of (1.4) is guaranteed for any fixed $\varepsilon$ (see Sakamoto [8;9]). Then since for $s \geqq T_{0}{ }^{\kappa}$ all the coefficients of $\left\{\tilde{P}_{\varepsilon}, \tilde{B}_{k \varepsilon}\right\}$ are constant, considering especially the hyperbolic mixed problem (1.4) with initial data $D_{t}^{j} u_{\varepsilon}\left(T_{0}^{\kappa}, x, y\right)(j=0, \ldots, m-1)$ in $\left(T_{0}^{\kappa}, \infty\right) \times \mathbb{R}_{+}^{n}$, we have the solution $u_{\varepsilon}$ of (1.4) such that $e^{-\gamma t} u_{\varepsilon} \in H_{m-2+r}\left(\mathbb{R}_{+} \times \mathbb{R}_{+}^{n}\right)$ for $\gamma>\gamma_{0}$ where $\gamma_{0}$ is some positive constant independent of $\varepsilon_{0}$ To prove the existence theorem of (1.2) we apply the following energy inequality which will be established in the later section.

Theorem 1.1. Let $\nu$ be a non-negative integer. Then there are a constant $G$ and an integer $\tilde{N}$ such that for any solution $u_{\varepsilon}$ of (1.4) the following inequality holds:

$$
\begin{aligned}
\left\|u_{\varepsilon}\right\|_{H_{\nu+m-1}\left(\left(0, T_{1}\right) \times R_{+}^{n}\right)}^{2} \leqq C\left\{\left\|f_{1}\right\|_{H_{\nu+\tilde{N}+1}\left(\left(0, T_{1}\right) \times R_{+}^{n}\right)}^{2}\right. & \\
& +\sum_{j=1}^{m_{+}}|| g_{j 1} \|_{H_{m-1-r_{j}+\tilde{N}+1+\nu}}^{2}\left(\left(0, T_{1}\right) \times R^{n-1}\right)
\end{aligned}
$$

where $G$ and $\tilde{N}$ are both independent of $\varepsilon \geqq 0$.

Now a family of solutions $\left\{u_{\varepsilon}\right\}_{\varepsilon \leq \varepsilon_{0}}$ forms a bounded set in the space $H_{m-1+\nu}\left(\left(0, T_{1}\right) \times \mathbb{R}_{+}^{n}\right)$ since the right hand side of $(1.5)$ is independent of $\varepsilon_{\text {. Th }}$ Thus there exist a sequence $\left\{u_{\varepsilon_{k}}\right\}_{k=1,2, \ldots}$ and a function $u \in H_{\nu+m-1}\left(\left(0, T_{1}\right) \times \mathbb{R}_{+}^{n}\right)$ such that $u_{\varepsilon_{k}} \rightarrow u$ weakly in $H_{\nu+m-1}\left(\left(0, T_{1}\right) \times \mathbb{R}_{+}^{n}\right)$ as $k \rightarrow \infty$. Since for each $k u_{\varepsilon_{k}}$ is the solution of the problem (1.4), making $k \rightarrow \infty$, we observe $\tilde{P}[u]=f_{1}$ and $D_{t}^{j} u(0, x, y)=0(j=1, \ldots$, $m-1)$ respectively by usual arguments.

Next we shall show $\tilde{B}_{j}[u]=g_{j 1}\left(j=1, \ldots, m_{+}\right)$according to Kimura [2]. For $\phi(s, y) \in \mathscr{D}\left(\left(0, T_{1}\right) \times \boldsymbol{R}^{n-1}\right)$, we get

$$
\begin{aligned}
& \int_{0}^{T_{1}} \int_{R^{n-1}} \tilde{B}_{j \varepsilon}\left[u_{\varepsilon}\right](s, y) \phi(s, y) d y d s \\
& \quad=-\int_{0}^{T_{1}} \int_{R^{n-1}} \int_{0}^{1} \partial_{x}\left(\chi(x) \tilde{B}_{j \varepsilon}\left[u_{\varepsilon}\right]\right) \phi(s, y) d x d y d s
\end{aligned}
$$




$$
=-\int_{0}^{T_{1}} \int_{R^{n-1}} \int_{0}^{1}\left\{\partial_{x}\left(\chi(x) \tilde{B}_{j \varepsilon}\right)\left[u_{\varepsilon}\right]+\chi(x) \tilde{B}_{j \varepsilon}\left[\partial_{x} u_{\varepsilon}\right]\right\} \phi(s, y) d x d y d \mathrm{~s},
$$

where $\chi(x) \in C^{\infty}\left(\boldsymbol{R}_{+}\right)$is equal to 1 in a neighborhood of $x=0$ and vanishes when $x>1$. Taking account of $\tilde{B}_{j \varepsilon}\left[u_{\varepsilon}\right]=g_{j 1}\left(j=1, \ldots, m_{+}\right)$, we have as $\varepsilon \rightarrow 0$,

$$
\begin{aligned}
& \int_{0}^{T_{1}} \int_{R^{n-1}} g_{j 1}(s, y) \phi(s, y) d y d s \\
& \quad=-\int_{0}^{T_{1}} \int_{R^{n-1}} \int_{0}^{1}\left\{\partial_{x}\left(\chi \tilde{B}_{j}\right)[u]+\tilde{B}_{j}\left[\partial_{x} u\right]\right\} \phi(s, y) d x d y d s \\
& \quad=-\int_{0}^{T_{1}} \int_{R^{n-1}} \int_{0}^{1} \partial_{x}\left(\chi \tilde{B}_{j}[u]\right) \phi(s, y) d x d y d s \\
& \quad=\int_{0}^{T_{1}} \int_{R^{n-1}} \tilde{B}_{j}[u] \phi(s, y) d y d s
\end{aligned}
$$

which concludes $\tilde{B}_{j}[u]=g_{j 1}$ in $\mathscr{D}^{\prime}\left(\left(0, T_{1}\right) \times \boldsymbol{R}^{n-1}\right), j=1, \ldots, m_{+}$. Therefore we see that $u$ satisfies (1.2). Then the uniqueness of solution follows from (1.5) for $\varepsilon=0$.

\section{§2. Singular integral operators with a parameter $\gamma>0$}

For a multi-index $\alpha=\left(\alpha_{0}, \alpha_{1}, \ldots, \alpha_{n-1}\right)=\left(\alpha_{0}, \alpha^{\prime}\right)$, we write $D_{t y}^{\alpha}=$ $D_{t}^{\alpha_{0}} D_{y_{1}}^{\alpha_{1}} \ldots D_{y_{n-1}}^{\alpha_{n-1}}$. Let $\Omega$ be either $\boldsymbol{R}_{+}^{n}$ or $\boldsymbol{R}^{n-1}$. We define, for $I$ an open interval in $t, \mathscr{H}_{m,-r}(I \times \Omega)$ with a positive parameter $\gamma$ as follows. $u \in \mathscr{H}_{m,-r}(I \times \Omega)$ if and only if $e^{-r t} u$ belongs to $H_{m}(I \times \Omega)$ with the inner product defined respectively by

i) in the case for $\Omega=\boldsymbol{R}_{+}^{n}$

$$
\begin{aligned}
(f, g)_{\mathscr{H}_{m,-r}(I \times \Omega)} & =\sum_{i+|\nu| \leqq m} \int_{I \times \Omega} e^{-2 \gamma t} D_{t y}^{\nu} D_{x}^{i} f \overline{D_{t y}^{\nu} D_{x}^{i g}} d t d x d y \\
\langle f, g\rangle_{\mathscr{H}_{m,-\gamma}(I \times \partial \Omega)} & =\sum_{|\nu| \leqq m} \int_{I \times \partial \Omega} e^{-2 \gamma t} D_{t y}^{\nu} f \overline{D_{t y}^{\nu} g} d t d y .
\end{aligned}
$$

ii) in the case for $\Omega=\boldsymbol{R}^{n-1}$,

$$
(f, g)_{\mathscr{H}_{m,-\gamma}(I \times \Omega)}=\sum_{|\nu| \leqq m} \int_{I \times \Omega} e^{-2 \gamma t} D_{t y}^{\nu} f \overline{D_{t y}^{\nu} g} d t d y .
$$

For the both cases the norm are denoted by

$$
\|f\|_{\mathscr{H}_{m,-r}}^{2}{ }_{(I \times \Omega)}=(f, f)_{\mathscr{H}_{m,-r}}{ }_{\left.m_{1} \times \Omega\right)}
$$




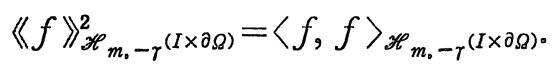

In this section and $\S 3$ we treat the case ii) only. We denote there $\mathscr{H}_{m,-\gamma}\left(\boldsymbol{R} \times \mathbb{R}^{n-1}\right)$ by $\mathscr{H}_{m,-r}$. In $\S 4$ the case i) is treated。 Then we denote $(f, g)_{\mathscr{H}_{m,-\gamma}\left(R \times R_{+}^{n}\right)}$ and $\langle f, g\rangle_{\mathscr{H}_{m,-\gamma}\left(\boldsymbol{R} \times R^{n-1}\right)}$ by $(f, g)_{\mathscr{H}_{m,-r}}$ and $\langle f, g\rangle_{\mathscr{H}_{m_{-}-r}}$ respectively. In particular for the both cases we denote $\mathscr{H}_{0,-r}$ by $\mathscr{H}_{-r}$ simply.

We say that $a(t, y ; \gamma, \tau, \eta)$ is a regular symbol of degree $m$ if it satisfies the conditions:

i) $a$ is homogeneous of degree $m$ in $(\gamma, \tau, \eta) \in \mathbb{R}_{+} \times \mathbb{R}^{n}$.

ii) $a$ is infinitely differentiable in $(t, y ; \gamma, \tau, \eta) \in \mathbb{R}^{n} \times \mathbb{R}_{+} \times \mathbb{R}^{n}$ and satisfies

$$
\sup _{\substack{(t, y) \in R^{n} \\(\gamma, \tau, \eta) \in L^{+}}}\left|D_{t y}^{\alpha} D_{\tau}^{i} D_{\tau \eta}^{\beta} a(t, y ; \gamma, \tau, \eta)\right|<\infty,
$$

where $L^{+}=\left\{(\gamma, \tau, \eta) \in S^{n} \mid \gamma>0\right\}$.

Then we define a singular integral operator for a regular symbol $a$

$$
\begin{aligned}
& a\left(t, y ; \gamma, D_{t}, D_{y}\right) u \\
& \quad=(2 \pi)^{-n} e^{\gamma t} \int_{R^{n}} e^{i(t, y) \cdot(\tau, \eta)} a(t, y ; \gamma, \tau, \eta) \widehat{e^{-\gamma t} u}(\tau, \eta) d \tau d \eta
\end{aligned}
$$

for $u \in C_{0}\left(\mathbb{R}^{n}\right)$, following to Sakamoto [8;9]. When we regard $a(t, y ; \gamma, \tau, \eta)$ as a pseudo-differential operator for any fixed $\gamma>0$, $\Lambda(\gamma, \tau, \eta)=\left(\gamma^{2}+\tau^{2}+|\eta|^{2}\right)^{1 / 2}$ essentialy play the same role as a weight function. Therefore the same arguments as established for the pseudodifferential operator with a weight function hold for $a\left(t, y ; \gamma, D_{t}, D_{y}\right)$, too. We represent the symbol of a singular integral operator $a(t, y$; $\left.\gamma, D_{t}, D_{y}\right)$ by $\sigma(a)$. Then the following results hold for $a\left(X^{\prime}\right)$ where $X^{\prime}=(t, y ; \gamma, \tau, \eta)$ (see Kumano-go [3]).

Lemma A. Let $a\left(X^{\prime}\right)$ be a regular symbol of degree $k$. Then for any real number $s$

i) $\sigma\left(a\left(t, y ; \gamma, D_{t}, D_{y}\right) \Lambda^{s}\left(\gamma, D_{t}, D_{y}\right)\right)=a\left(X^{\prime}\right) \Lambda^{s}(\gamma, \tau, \eta)$,

ii) for a non-negative integer $m$, there holds

$$
\begin{aligned}
& \sigma\left(a\left(t, y ; \gamma, D_{t}, D_{y}\right) \Lambda^{s}\left(\gamma, D_{t}, D_{y}\right) b(t)^{m}\right) \\
& \quad=\sum_{i=0}^{m}\left\{D_{t}^{i} b(t)^{m} / i !\right\}\left\{(\partial / \partial \tau)^{i}\left(a\left(X^{\prime}\right) \Lambda^{s}(\gamma, \tau, \eta)\right)\right\}+\hat{a}\left(X^{\prime}\right),
\end{aligned}
$$

where $\Lambda^{-(s-m-1)-k} \hat{a}\left(X^{\prime}\right) \in S_{10}{ }^{0}$. 
From Lemma A it follows immediately

Lemma B. There exists a constant $C$ such that for any $u \in \mathscr{H}_{s-1+k_{,}-r}$ it holds

$$
\left\|\left[a\left(t, y ; \gamma, D_{t}, D_{y}\right) \Lambda^{s}, b(t)\right] u\right\|_{\mathscr{H}_{-\gamma}} \leqq C\|u\|_{\mathscr{H}_{s-1+k,-\gamma}} .
$$

We introduce the norm of $a(x) \in \mathscr{B}_{\alpha}\left(\boldsymbol{R}^{n}\right)$ :

$$
\begin{aligned}
& |a|_{\mathscr{B}_{\alpha}\left(R^{n}\right)}=\sum_{|\nu| \leq[\alpha]} \sup _{x \in R^{n}}\left|D_{x}^{\nu} a(x)\right| \\
& \quad+\sum_{|\nu|=[\alpha]} \sup _{x_{i} \in R^{n}(i=1,2)}\left|\left(D_{x}^{\nu} a\right)\left(x_{1}\right)-\left(D_{x}^{\nu} a\right)\left(x_{2}\right)\right| /\left|\left(x_{1}-x_{2}\right)\right|^{\alpha-[\alpha]} .
\end{aligned}
$$

Let us consider in detail the singular integral operator associated with a non-regular symbol with a parameter $\gamma>0$. Let $\Gamma$ be an open conic set in $\boldsymbol{R}^{l} \times \boldsymbol{R}^{n+1}$, that is, $\Gamma$ is an open subset in $\boldsymbol{R}^{l} \times \boldsymbol{R}^{n+1}$ and for any $(x, \theta) \in \Gamma$ and $s>0$ it holds $(x, s \theta) \in \Gamma$, and let $H(x ; \gamma, \tau, \eta)$ be homogeneous of degree 0 with respect to $(\gamma, \tau, \eta)$. Now according to [7] we introduce a symbol class for $\Gamma_{1}=\{(x ; \gamma, \tau, \eta)$ $\in \Gamma ;|(\gamma, \tau, \eta)|=1\}$

$$
\begin{aligned}
& \mathscr{B}_{\alpha, k}\left(\Gamma_{1}\right)=\left\{\left.H(x ; \gamma, \tau, \eta)|| H\right|_{\mathscr{H}_{\alpha, k}\left(\Gamma_{1}\right)}=\sum_{\substack{|\nu| \leq\{\alpha] \\
|\mu|+i \leq k}} \sup _{\Gamma_{1}}\left|H_{\mu \nu i}(x ; \gamma, \tau, \eta)\right|\right. \\
& \left.+\sum_{\substack{|\mu|=[\alpha] \\
|\nu|+i \leq k}} \sup _{\left.x_{j} ;, \tau, \eta\right) \in \Gamma_{1}, j=1,2} \frac{\left|H_{\mu \nu i}\left(x_{1} ; \gamma, \tau, \eta\right)-H_{\mu \nu i}\left(x_{2} ; \gamma, \tau, \eta\right)\right|}{\left|\left(x_{1}-x_{2}\right)\right|^{\alpha-[\alpha]}}<\infty\right\},
\end{aligned}
$$

where $H_{\mu \nu i}(x ; \gamma, \tau, \eta)=D_{x}^{\mu} D_{\tau \eta}^{\nu} D_{r}^{i} H(x ; \gamma, \tau, \eta)$.

We denote $\mathscr{B}_{\alpha, k}\left(\boldsymbol{R}^{n} \times L^{+}\right)$by $\mathscr{B}_{\alpha, k}$ and $\mathscr{B}_{\alpha}\left(\boldsymbol{R}^{n}\right)$ by $\mathscr{B}_{\alpha}$ for simplicity.

Let $Y_{l}(\gamma, \tau, \eta)$ be a normalized real spherical harmonics of degree $l$ such that $\int_{S^{n}} Y_{l}^{2}(\gamma, \tau, \eta) d \sigma=1$. Let us write a base of the spherical harmonics of $l$-th order as $\left\{Y_{l m}(\gamma, \tau, n)\right\}_{m=1,2, \ldots n(l)}$.

Let $H\left(X^{\prime}\right) \in \mathscr{R}_{\alpha, k}(\alpha \geqq 0)$. Then there exists an extension $\stackrel{\circ}{H}\left(X^{\prime}\right)$ of $H\left(X^{\prime}\right)$ such that $\stackrel{\circ}{H}\left(X^{\prime}\right)$ is homogeneous of degree 0 with respect to $(\gamma, \tau, \eta) \in \boldsymbol{R}^{n+1}$ and that $\stackrel{\circ}{H}\left(X^{\prime}\right)$ satisfies

$$
\left|H^{\circ}\right|_{\mathscr{R}_{\alpha, k}\left(R^{n} \times S^{n}\right)} \leqq C|H|_{\mathscr{H}_{\alpha, k}\left(R^{n} \times L^{+}\right)}
$$

(see [7]).

We put $a_{l m}(t, y)=\int_{S^{n}} \stackrel{\circ}{H}(t, y ; \gamma, \tau, \eta) Y_{l m}(\gamma, \tau, \eta) d \sigma_{\dot{\mathrm{s}}}$ Then we have 


$$
\stackrel{\circ}{H}\left(X^{\prime}\right)=a(t, y)+\sum_{l \geq 1, m} a_{l m}(t, y) Y_{l m}(\gamma, \tau, \eta) .
$$

Now we define the singular integral operator $H\left(t, y ; \gamma, D_{t}, D_{y}\right)$ with $H\left(X^{\prime}\right) \in \mathscr{B}_{\alpha, k}$ for $\alpha \geqq 0, k \in \mathbb{N}:$ for $u \in \mathscr{S}\left(\mathbb{R}^{n}\right)$,

$$
\begin{aligned}
& H\left(t, y ; \gamma, D_{t}, D_{x}\right) u(t, y)=\stackrel{\circ}{H}\left(t, y ; \gamma, D_{t}, D_{y}\right) u(t, y) \\
& \quad=\sum_{l \geq 0, m} e^{\gamma t} a_{l m}(t, y)(2 \pi)^{-n} \int_{R^{n}} e^{i(t, y) \circ(\tau, \eta)} Y_{l m}(\gamma, \tau, \eta) \widehat{e^{-\gamma t}} u(\tau, \eta) d \tau d \eta
\end{aligned}
$$

where we write $a_{0}(t, y)$ for $a(t, y)$ and $Y_{0}$ for the identity operator. We have

$$
\|H u\|_{\mathscr{H}_{-\gamma}} \leqq C|H|_{\mathscr{F}_{0,2 n}}|| u \|_{\mathscr{H}_{-\gamma}}
$$

By the mean value theorem

$$
H\left(X^{\prime}\right)=H^{0}\left(X^{\prime}\right)+\gamma H^{1}\left(X^{\prime}\right)
$$

where $H^{0}\left(x^{\prime}\right)=H(t, y ; 0, \tau, \eta)$ and $H^{1}\left(X^{\prime}\right)$ is homogeneous of degree -1 with respect to $(\gamma, \tau, \eta)$.

Proposition 0。 For constants $0<\alpha<1,0 \leqq \delta$ and $0<\mu$ assume that $c(t, y)$ satisfies the following properties:

$$
\left\{\begin{array}{l}
c(t, y)=c_{1}(t, y)+c_{2}(t, y) \\
\left|c_{i}(t, y)\right|_{\mathscr{B}_{0}}<\mu, \quad i=1,2 \\
\left|b(t) c_{2}(t, y)\right|_{\mathscr{B}_{1+\alpha}}<\delta \\
c_{1}(t, y) \in \mathscr{B}_{1+\alpha^{\circ}}
\end{array}\right.
$$

Then it holds for $u \in \mathscr{H}_{-r}, i=0, \ldots, n-1$ and $H\left(X^{\prime}\right) \in \mathscr{R}_{\alpha, k}$

$$
\left\|(c H-H c) D_{t y}^{e_{i}} b(t) u\right\|_{\mathscr{H}_{-\gamma}} \leqq C\left((1+\gamma \mu)\|b(t) u\|_{\mathscr{H}_{-\gamma}}+\delta !\|u\|_{\mathscr{H}_{-\gamma}}\right)
$$

where $e_{i}=\left(0, \ldots,, \check{i}_{1}, \ldots, 0\right)$ and $h$ is a sufficiently large integer.

The proof consists of two parts. We begin with the proof for the case when $H\left(X^{\prime}\right)$ is independent of $(t, y)$. We shall prepare two lemmas.

Lemma 2. 1. There exists a constant $C$ such that for $u \in \mathscr{H}_{-r}$,

i) $\quad r\left\|H^{1}\left(\gamma, D_{t}, D_{y}\right) u\right\|_{\mathscr{H}_{-\gamma}} \leqq C\|u\|_{\mathscr{H}_{-\gamma}}$,

ii) $\gamma\left\|H_{j}^{1}\left(\gamma, D_{t}, D_{y}\right) c(t, y) u\right\|_{\mathscr{H}-\gamma}, \gamma\left\|c(t, y) H_{j}^{1}\left(\gamma, D_{t}, D_{y}\right) u\right\|_{\mathscr{H}}-\gamma$

$$
\leqq C \gamma \mu\|u\|_{\mathscr{H}_{-\gamma}}
$$


where $H_{j}^{1}(\gamma, \tau, \eta)=(\tau, \eta)^{e_{j}} H^{1}(\gamma, \tau, \eta)$ for $j=0, \ldots, n-1$.

Proof. i) By the definition (2.0)

$$
\begin{aligned}
& \gamma\left\|H^{1}\left(\gamma, D_{t}, D_{y}\right) u\right\|_{\mathscr{E}_{-\gamma}}=\gamma\left\|\mathscr{F}_{i r}^{-1}\left[H^{1}\right](\gamma, \tau, \eta) \widehat{e^{-r t} u}(\tau, \eta)\right\|_{L^{2}\left(\boldsymbol{R}^{n}\right)} \\
& =\gamma\left\|H^{1}(\gamma, \tau, \eta) \widehat{e^{-\gamma t} u}(\tau, \eta)\right\|_{L^{2}\left(\boldsymbol{R}^{n}\right)} \text { (Plancherel's formula) } \\
& \leqq\left|\gamma H^{1}\right|_{0}\|u\|_{\mathscr{H}_{-\gamma^{*}}}
\end{aligned}
$$

Since $\gamma H^{1}\left(X^{\prime}\right)$ is homogeneous of degree 0 in $(\gamma, \tau, \eta)$, we have i). ii) In the same way as in i)

$$
\gamma\left\|H_{j}^{1} c u\right\|_{\mathscr{H}_{-\gamma}} \leqq C \gamma\|c u\|_{\mathscr{H}_{-r}} \leqq C \gamma \mu\|u\|_{\mathscr{H}_{-r}}
$$

and similarly

$$
\gamma\left\|c H_{j}^{1} u\right\|_{\mathscr{H}_{-\gamma}} \leqq \gamma \mu\left\|H_{j}^{1} u\right\|_{\mathscr{H}_{-\gamma}} \leqq C \gamma \mu\|u\|_{\mathscr{H}_{-\gamma}} .
$$

Lemma 2.2. There exists a constant $C$ such that for $|\beta|=1$ and $u \in \mathscr{H}_{-r}$, we have

i) $\quad\left\|\left(c H^{0}-H^{0} c\right) D_{t y}^{\beta}(b(t) u)\right\|_{\mathscr{H}_{-\gamma}} \leqq C\left(\|b(t) u\|_{\mathscr{H}_{-\gamma}}+\delta\|u\|_{\mathscr{H}_{-\gamma}}\right)$.

ii) $\quad \gamma\left\|\left(c H^{1}\right) D_{t y}^{\beta}(b(t) u)\right\|_{\mathscr{H}_{-\gamma}}, \gamma\left\|\left(H^{1} c\right) D_{t y}^{\beta}(b(t) u)\right\|_{\mathscr{H}_{-\gamma}}$

$$
\leqq C\left((1+\gamma \mu)\|b(t) u\|_{\mathscr{H}_{-\gamma}}+\delta\|u\|_{\mathscr{H}_{-r}}\right) .
$$

Proof. We write $z=(t, y)$ and $b(t)$ by $t$ for simplicity.

i) Put $D_{\varepsilon}=\left\{\left(z, z^{\prime}\right) \in \boldsymbol{R}^{2(n+1)}||\left(z-z^{\prime}\right) \mid>\varepsilon\right\}$ for $\varepsilon>0$. Then we consider the expression according to Calderón-Zygmund [1],

$$
\begin{aligned}
& e^{-\gamma z_{0}}\left(c H^{0}-H^{0} c\right) D_{z}^{\beta}\left(z_{0} u\right) \\
& =\lim _{\varepsilon \rightarrow 0} \int_{D_{\varepsilon}}\left(c_{2}(z)-c_{2}\left(z^{\prime}\right)\right) Y\left(z-z^{\prime}\right) D_{z^{\prime}}^{\beta}\left(e^{-\gamma z_{0}^{\prime}} z_{0}^{\prime} u\right) d z^{\prime} \\
& \quad+e^{-\gamma z_{0}}\left(c_{1} H^{0}-H^{0} c_{1}\right) D_{z}^{\beta}\left(z_{0} u\right),
\end{aligned}
$$

where $Y(z)$ is the kernel of the operator $H^{0}$, homogeneous of degree $-n$ and belongs to $\mathscr{E}\left(\boldsymbol{R}^{n} \backslash\{0\}\right)$ and its mean value over the sphere $|z|=1$ is zero. By integrating by parts we have

$$
e^{-\gamma z_{0}}\left(c H^{0}-H^{0} c\right) D_{z}^{\beta}\left(z_{0} u\right)=e^{-\gamma z_{0}}\left(c_{1} H^{0}-H^{0} c_{1}\right) D_{z}^{\beta}\left(z_{0} u\right)
$$




$$
\left\{\begin{array}{l}
+\lim _{\varepsilon \rightarrow 0}\left\{\int_{D_{\varepsilon}} Y\left(z-z^{\prime}\right)\left(D_{z^{\prime}}^{\beta} c_{2}\left(z^{\prime}\right)\right) e^{-\gamma z_{0}^{\prime}} z_{0}^{\prime} u d z^{\prime}\right. \\
+\int_{D_{\varepsilon}}\left(D_{z}^{\beta} Y\right)\left(z-z^{\prime}\right) e^{-\gamma z_{0}^{\prime}}\left(c_{2}(z)-c_{2}\left(z^{\prime}\right)\right) z_{0}^{\prime} u d z^{\prime} \\
\left.+\int_{\left|\left(z-z^{\prime}\right)\right|=\varepsilon} Y\left(z-z^{\prime}\right)\left(c_{2}(z)-c_{2}\left(z^{\prime}\right)\right) e^{-\gamma z_{0}^{\prime}} z_{0}^{\prime} u \beta_{i} d \sigma\right\}
\end{array}\right.
$$

where the last integral is extended over the surface of the sphere $\left|\left(z-z^{\prime}\right)\right|=\varepsilon$ and $\beta_{i}$ is the $i$-th direction cosine of the normal to the spherical surface.

Calderón-Zygmund [1] proved for $u \in L^{2}\left(\mathbb{R}^{n}\right)$

$$
\left\|(c T-T c) D_{z}^{\beta} u\right\|_{L^{2}\left(R^{n}\right)} \leqq C\|u\|_{L^{2}\left(R^{n}\right)}
$$

where $c(z) \in \mathscr{B}_{1+\alpha}\left(\mathbb{R}^{n}\right)$ and $T$ is an arbitrary singular integral operator with symbol independent of $z$. Since $c_{1}(z) \in \mathscr{B}_{1+\alpha}$, we have immediately

$$
\left\|\left(c_{1} H^{0}-H^{0} c_{1}\right) D_{z}^{\beta}\left(z_{0} u\right)\right\|_{\mathscr{H}_{-r}} \leqq C\left\|z_{0} u\right\|_{\mathscr{H}_{-r^{\circ}}}
$$

Observing that $\left|z_{0}^{\prime} D_{z}^{\beta}, c_{2}\left(z^{\prime}\right)\right|<$ const. $\delta$, by Theorem 2 in [1] we see that the first term of $(2.7)$ represents a function of $z$ whose $L^{2}$-norm does not exceed const. $\delta\|u\|_{\mathscr{H}_{-}}$. $^{\circ}$

Since $z_{0}^{\prime}=-\left(z-z^{\prime}\right)_{0}+z_{0}$, we have the second term of (2.7)

$$
\begin{aligned}
= & \int_{D_{\varepsilon}}-\left(z-z^{\prime}\right)_{0}\left(D_{z}^{\beta} Y\right)\left(z-z^{\prime}\right) e^{-\gamma z_{0}^{\prime}} c_{2}(z) u d z^{\prime} \\
& +\int_{D_{\varepsilon}}\left(z_{0} c_{2}(z)-z_{0}^{\prime} c_{2}\left(z^{\prime}\right)\right)\left(D_{z}^{\beta} Y\right)\left(z-z^{\prime}\right) e^{-\gamma z_{0}^{\prime}} u d z^{\prime} \\
= & I_{1}+I_{2} .
\end{aligned}
$$

Taking account of $\left|z_{0} c_{2}(z)\right|_{\mathscr{F}_{1+\alpha}}<\delta$, we have

$$
z_{0} c_{2}(z)-z_{0}^{\prime} c_{2}\left(z^{\prime}\right)=\sum_{j=0}^{n-1}\left(z_{i}-z_{i}^{\prime}\right)\left(z_{0} c_{2}(z)\right)_{z_{i}}+b\left(z, z^{\prime}\right)
$$

where $\left|b\left(z, z^{\prime}\right)\right| \leqq C \delta\left|z-z^{\prime}\right|^{1+\alpha}$. Hence, by the same method as used in the second, third and fourth terms in (44) of [1], we have

$$
\left\|I_{2}\right\|_{L^{2}\left(R^{n}\right)} \leqq C \delta\|\| u \|_{\mathscr{H}_{-r^{*}}}
$$

Similarly, noting that $\left|c_{2}(z)\right|<$ const. $\delta$, we have

$$
\left\|I_{1}\right\|_{L^{2}\left(R^{n}\right)} \leqq C \delta\|\mid u\|_{\mathscr{H}_{-\gamma^{*}}}
$$

From (2.9) it follows 


$$
\begin{aligned}
& \left|\int_{\left|\left(z-z^{\prime}\right)\right|=\varepsilon} e^{-\gamma z_{0}^{\prime}}\left(c_{2}(z)-c_{2}\left(z^{\prime}\right)\right) z_{0}^{\prime} Y\left(z-z^{\prime}\right) u \beta_{i} d \sigma\right| \\
& \quad \leqq C \delta \int_{S^{n}} e^{-\gamma(-\varepsilon \omega+z)}|u(-\varepsilon \omega+z)|\left|\varepsilon^{n} Y(\varepsilon \omega)\right| d \sigma
\end{aligned}
$$

where $\omega=\left(z-z^{\prime}\right) / \varepsilon$. Since $\varepsilon^{n} Y(\varepsilon \omega)=Y(\omega)$, we see that as $\varepsilon$ tend to zero this term has a limit whose absolute value is dominated by const. $\delta\left|e^{-\gamma z} u(z)\right|$. Therefore we have

$$
\left\|\left(c_{2} H^{0}-H^{0} c_{2}\right) D_{z}^{\beta}\left(z^{0} u\right)\right\|_{\mathscr{H}_{-r}} \leqq C \delta\|u\|_{\mathscr{H}_{-r}} .
$$

Gathering these results we obtain i).

ii) Noting that $\left|z_{0}\left(D_{z}^{\beta} c\right) u\right| \leqq\left|\left(D_{z}^{\beta} c_{1}\right) z_{0} u\right|+\mid z_{0}\left(D_{z}^{\beta} c_{2}\right) u \leqq C\left(\left|z_{0} u\right|+\delta|u|\right)$, the proof of ii) is carried out using Lemma 2.1 .

The proof of Proposition 0. By combining i) with ii) of Lemma 2.2 we easily see that it holds for $i=0, \ldots, n-1$,

$$
\begin{aligned}
& \left\|\left(c(t, y) H\left(\gamma, D_{t}, D_{y}\right)-H\left(\gamma, D_{t}, D_{y}\right) c(t, y)\right) D_{t y}^{e_{i}}(t u)\right\|_{\mathscr{H}_{-\gamma}} \\
& \quad \leqq C\left((1+\gamma \mu)\|t u\|_{\mathscr{H}_{-\gamma}}+\delta\|u\|_{\mathscr{H}_{-\gamma}}\right) .
\end{aligned}
$$

Next we consider the case where $H\left(X^{\prime}\right)$ depends on $(t, y)$. Let expand $H\left(X^{\prime}\right)$ in spherical harmonics in (2. 6) and apply the above estimate to every term of the numerical series. Then by the well known properties about $\left\{Y_{l m}\right\}$ we see that they are dominated in $\mathscr{H}_{-\gamma}$-norm by a convergent numerical series (see [1][5]). Therefore we have Proposition 0 .

Remark 2. In case $c_{2}=0$, by the same arguments as in the above for $c_{2}=0$ we have

$$
\left\|(c H-H c) D_{t y}^{\beta}(b(t) u)\right\|_{\mathscr{H}_{-r}} \leqq C\left((1+\gamma \mu)\|b(t) u\|_{\mathscr{H}_{-\gamma}}\right)
$$

which corresponds to the conclusion of our Proposition for $\delta=0$.

\section{§3. The Estimates for Singular Integral Operators with Some Non-regular Symbols}

Now we consider a non-regular symbol $H\left(X^{\prime}\right)$ of the form: 
$(* *) \quad \begin{cases}\text { i) } & H\left(X^{\prime}\right)=\left(H_{r}+H_{\mu}+H_{\delta}\right)\left(X^{\prime}\right), \\ \text { ii) } & \left|H_{\mu}\right|_{\mathscr{B}_{0,2 h},}\left|H_{\delta}\right|_{\mathscr{B}_{0,2 h}}<\mu, \\ \text { iii) } & \left|b(t) H_{\delta}\right|_{\mathscr{B}_{1+\alpha, 2 h}}<\delta, \\ \text { iv) } & H_{\mu}\left(X^{\prime}\right) \in \mathscr{B}_{1+\alpha, 2 h}\end{cases}$

where $H_{\mu}\left(X^{\prime}\right)$ and $H_{\delta}\left(X^{\prime}\right)$ are non-regular symbols of degree 0 with $0<\mu \leqq \delta<c_{0}<\infty$ and $H_{r}$ is a regular symbol, and $0<\alpha<1, h \in \mathbb{N}$. Actually it will be shown in $\S 4$ that such a non-regular symbol does exist. We assume $h$ is chosen appropriately large in the following argument.

Lemma 3.1. For any $H_{1}\left(X^{\prime}\right) \in \mathscr{B}_{\alpha, 2 h}(0 \leqq \alpha<1)$ and any $k \in \mathbb{N}$, there is a constant $C$ such that for $u \in \mathscr{H}_{k-1,-r}$

$$
\begin{aligned}
& \left\|\left(H_{1} \circ H-H_{1} H\right) \Lambda^{k} b(t) u\right\|_{\mathscr{H}_{-\gamma}} \\
& \quad \leqq C\left((1+\gamma \mu)\|b(t) u\|_{\mathscr{H}_{k-1,-\gamma}}+\delta\|u\|_{\mathscr{H}_{k-1,-\gamma}}\right)
\end{aligned}
$$

where $H_{1} \circ H\left(t, y ; \gamma, D_{t}, D_{y}\right)$ is a singular integral operator with symbol $H_{1}\left(X^{\prime}\right) H\left(X^{\prime}\right)$.

Proof. We write $b(t)$ by $t$ for simplicity. If $H\left(X^{\prime}\right)$ is a regular symbol, by the representation $\stackrel{\circ}{H}_{1}$ given in $(2.2)$ we have

$$
\begin{aligned}
& \left\|\left(H_{1} \circ H-H_{1} H\right) \Lambda^{k} t u\right\|_{\mathscr{H}_{-r}} \\
& \quad \leqq \sum_{l, m}\left|a_{l m}\right|\left\|\left(Y_{l m} \circ H-Y_{l m} H\right) \Lambda^{k} t u\right\|_{\mathscr{H}_{-\gamma}} \\
& \leqq \text { const. }\|t u\|_{\mathscr{H}_{k-1,-r}}(\operatorname{see}[8 ; 9]) 。
\end{aligned}
$$

Therefore it suffices to prove (3.1) for the case where $H\left(X^{\prime}\right)=$ $H_{\mu}\left(X^{\prime}\right)+H_{\delta}\left(X^{\prime}\right)$. By the expansion formula we get

$$
\stackrel{\circ}{H}_{\mu}\left(X^{\prime}\right)=\sum_{l, m} a_{l m}^{\mu}(t, y) Y_{l m}(\gamma, \tau, \eta)
$$

and

$$
\stackrel{\circ}{H}_{\delta}\left(X^{\prime}\right)=\sum_{l, m} a_{l m}^{\delta}(t, y) Y_{l m}(\gamma, \tau, \eta)
$$

We note that from (**)-ii), iii) and iv) in the above it follows that

$$
\left\{\begin{array}{l}
\text { ii })^{\prime}\left|a_{l m}^{\mu}(t, y)\right|_{\mathscr{B}_{0}},\left|a_{l m}^{\delta}(t, y)\right|_{\mathscr{B}_{0}} \leqq C \mu l^{-2 h+(n / 2)} \\
\text { iii)' }\left|t a_{l m}^{\delta}(t, y)\right|_{\mathscr{B}_{1+\alpha}} \leqq C \delta l^{-2 h+(n / 2)} \\
\text { iv })^{\prime}\left|a_{l m}^{\mu}(t, y)\right|_{\mathscr{B}_{1+\alpha}} \leqq C l^{-2 h+(n / 2)}
\end{array}\right.
$$


(see [1] [5]).

Let $R_{i}(\gamma, \tau, \eta)=(\tau, \eta)_{i} / \Lambda(\gamma, \tau, \eta)(i=0, \ldots, n-1)$

and $R_{r}(\gamma, \tau, \eta)=\gamma / \Lambda(\gamma, \tau, \eta)$.

First we shall prove (3.1) for $k=1$. We have

$$
\begin{aligned}
& \left\|\left(H_{1} \circ \stackrel{\circ}{H}_{\delta}-H_{1} \stackrel{\circ}{H}_{\delta}\right) \Lambda t u\right\|_{\mathscr{C}} \\
& =\sum_{l, m}\left\|\sum_{i=0}^{n-1}\left(a_{l m}^{\delta} H_{1}-H_{1} a_{l m}^{\delta}\right) D_{t y}^{e_{i}} Y_{l m} R_{i} t u+\left(a_{l m}^{\delta} H_{1}-H_{1} a_{l m}^{\delta}\right) \gamma\left(Y_{l m} R_{\gamma} t u\right)\right\|_{\mathscr{C}}{ }_{-\gamma} \\
& \leqq C \gamma \mu\|t u\|_{\mathscr{H}_{-\gamma}} \\
& +\sum_{l, m} \sum_{i=0}^{n-1}\left\{\|\left(a_{l m}^{\delta} H_{1}-H_{1} a_{l m}^{\delta}\right) D_{t y}^{e_{i}}\left(\left[\left[Y_{l m} R_{i}, t\right] u\right) \|_{\mathscr{H}_{-r}}\right.\right. \\
& \text { (3.3) } \left.\quad+\left\|\left(a_{l m}^{\delta} H_{1}-H_{1} a_{l m}^{\delta}\right) D_{t y}^{e_{i}}\left(t Y_{l m} R_{i} u\right)\right\|_{\mathscr{H}_{-\gamma}}\right\} \text {. }
\end{aligned}
$$

Lemma B implies that (3.2) is dominated by const. $\delta\|u\|_{\mathscr{C}_{-r^{*}}}$ By using (2.10) (3.3) is dominated by const. $\delta\|u\|_{\mathscr{H}_{-}}$.

Therefore we have

$$
\left\|\left(H_{1} \circ H_{\delta}-H_{1} H_{\delta}\right) \Lambda t u\right\|_{\mathscr{H}_{-\gamma}} \leqq C\left(\gamma \mu\|t u\|_{\mathscr{H}_{-\gamma}}+\delta\|u\|_{\mathscr{H}_{-\gamma}}\right) .
$$

On the other hand, since $H_{\mu}\left(X^{\prime}\right) \in \mathscr{B}_{1+\alpha, 2 h}\left(\boldsymbol{R}^{n}\right)$ on account of Remark 2, (**)-ii)' and $i \mathrm{v})^{\prime}$ we have similarly

$$
\left\|\left(H_{1} H_{\mu}-H_{1} \circ H_{\mu}\right) \Lambda t u\right\|_{\mathscr{H}_{-\gamma}} \leqq C(1+\gamma \mu)\|t u\|_{\mathscr{H}}{ }_{-\gamma^{\circ}}
$$

Combining (3.4) and (3.5) we get

$$
\begin{array}{r}
\left\|\left(H_{1} \circ\left(H_{\mu}+H_{\delta}\right)-H_{1}\left(H_{\mu}+H_{\delta}\right)\right) \Lambda t u\right\|_{\mathscr{H}_{-\gamma}} \leqq C\left((1+\gamma \mu)\|t u\|_{\mathscr{H}_{-\gamma}}\right. \\
\left.+\delta\|u\|_{\mathscr{H}_{-\gamma}}\right) .
\end{array}
$$

When $k \geqq 2$, we have

$$
\begin{aligned}
\| & \left(H_{1} \circ\left(H_{\mu}+H_{\delta}\right)-H_{1}\left(H_{\mu}+H_{\delta}\right)\right) \Lambda^{k} t u \|_{\mathscr{H}_{-r}} \\
& \leqq \\
\quad & +\left\|\left(H_{1} \circ H_{\delta}-H_{1} H_{\delta}\right) \Lambda\left[\Lambda^{k-1}, t\right] u\right\|_{\mathscr{H}_{-\gamma}} \\
& \left.+\|\left(H_{1} \circ H_{\mu}-H_{1} H_{\delta}\right) \Lambda t \Lambda_{\mu}\right) \Lambda\left(\Lambda^{k-1} u \|_{\mathscr{H}_{-r}} t u\right) \|_{\mathscr{H}_{-r}}
\end{aligned}
$$

applying Lemma B to the first term of (3.7) and by (3.4) and (3.5) we have

$$
\leqq C\left((1+\gamma \mu)\|t u\|_{\mathscr{H}_{k-1,-\gamma}}+\gamma\|u\|_{\mathscr{H}_{k-1,-\gamma}}\right) .
$$


Hence we complete the proof of Lemma 3.1.

Lemma 3.2. For $u \in \mathscr{H}_{k-1,-\gamma}$ there exists a constant $C$ such that

$$
\left\|\left(\bar{H}-H^{*}\right) \Lambda^{k} b(t) u\right\|_{\mathscr{H}_{-\gamma}} \leqq C\left((1+\gamma \mu)\|b(t) u\|_{\mathscr{H}_{k-1,-\gamma}}+\delta\|u\|_{\mathscr{H}_{k-1,-\gamma}}\right),
$$

where $H^{*}$ is a formal adjoint operator of $H$ with respect to $(\tau, \eta)$ and $\bar{H}$ is the singular integral operator with symbol $\bar{H}\left(X^{\prime}\right)$.

Proof. It is sufficient to prove for $H=H_{\mu}+H_{\delta}$ by $[8 ; 9]$. The proof for $k=1$ is done as follows. By expanding the function $\stackrel{\circ}{H}_{\mu}+\stackrel{\circ}{H}_{\delta}$ in spherical harmonics, we have

$$
\begin{aligned}
& \left\|\left(\bar{H}_{\mu}+\bar{H}_{\delta}-H_{\mu}^{*}-H_{\delta}^{*}\right) A b(t) u\right\|_{\mathscr{H}_{-\gamma}} \\
& \quad \leqq \sum_{l, m}\left\{\sum_{i=0}^{n-1} \|\left(Y_{l m}\left(\bar{a}_{l m}^{\mu}+\bar{a}_{l m}^{\delta}\right)-\left(\bar{a}_{l m}^{\mu}+\bar{a}_{l m}^{\delta}\right) Y_{l m}\right) D_{t y}^{e_{i}} R_{i} b(t) u\right) \|_{\mathscr{H}} \\
& \left.\quad+\left\|\left(Y_{l m}\left(\bar{a}_{l m}^{\mu}+\bar{a}_{l m}^{\delta}\right)-\left(\bar{a}_{l m}^{\mu}+\bar{a}_{l m}^{\delta}\right) Y_{l m}\right) \gamma R_{r}(b(t) u)\right\|_{\mathscr{H}_{-\gamma}}\right\} \\
& \quad \leqq C\left((1+\gamma \mu)\|b(t) u\|_{\mathscr{H}_{-\gamma}}+\delta \|\left. u\right|_{\mathscr{H}_{-\gamma}}\right) \text { in the same manner as in }
\end{aligned}
$$

deriving (3.6) in Lemma 3.1.

The case for $k \geqq 2$ is carried out as in Lemma 3.1.

Lemma 3. 3. Let $\mathscr{H} \in \mathscr{B}_{k, 2 h} \cap \mathscr{B}_{1+\alpha, 2 h}$ and assume $\mid \mathscr{H}_{\mathscr{B}_{0,2 h}}<\mu$. Then for $u \in \mathscr{H}_{k-1,-\tau}$

i) $\quad\left(\| \mathscr{H} \Lambda^{k}-\Lambda^{k} \mathscr{H}\right) u\left\|_{\mathscr{H}-\gamma} \leqq C(1+\gamma \mu)\right\| u \|_{\mathscr{H}_{k-1,-\gamma}}$.

ii) $\quad\left\|\left(\mathscr{H}^{*} \Lambda^{k}-\Lambda^{k} \mathscr{H}^{*}\right) u\right\|_{\mathscr{H}_{-\gamma}} \leqq C(1+\gamma \mu)\|u\|_{\mathscr{H}_{k-1,-\gamma^{*}}}$

Proof. In case $k$ is even, we obtain for

$$
\Lambda^{k}\left(\gamma, D_{t}, D_{y}\right)=\left(\gamma^{2}+D_{t}^{2}+\sum_{i=1}^{n-1} D_{y_{i}}^{2}\right)^{k / 2}
$$

we obtain

$$
\left\|\left(\mathscr{H} \Lambda^{k}-\Lambda^{k} \mathscr{H}\right) u\right\|_{\mathscr{H}_{-\gamma}} \leqq C\|\| u \|_{\mathscr{H}_{k-1,-\gamma}}
$$

For $k=1$ we have

$$
\Lambda \mathscr{H}-\mathscr{H} \Lambda=\sum_{i=0}^{n-1}\left\{R_{i}\left(D_{t y}^{e_{i}} \mathscr{H}\right)+\left(R_{i} \mathscr{H}-\mathscr{H} R_{i}\right) D_{t y}^{e_{i}}\right\}+\left(R_{r} \mathscr{H}-\mathscr{H} R_{\tau}\right) \gamma_{0}
$$

The same method used in deriving (3.5) in Lemma 3.1. leads to 


$$
\|(\mathscr{H} \Lambda-\Lambda \mathscr{H}) u\|_{\mathscr{H}_{-r}} \leqq G .(1+\gamma \mu)\|u\|_{\mathscr{H}_{-r^{*}}}
$$

Also in case $k$ is odd ( $\geqq 3$ ), making use of (3.8), (3.9) we have

$$
\begin{aligned}
\left\|\left(\mathscr{H} \Lambda^{k}-\Lambda^{k} \mathscr{H}\right) u\right\|_{\mathscr{H}_{-r}} & \leqq\left\|(\mathscr{H} \Lambda-\Lambda \mathscr{H}) \Lambda^{k-1} u\right\|_{\mathscr{H}_{-r}}+C\|u\|_{\mathscr{H}_{k-1,-r}} \\
& \leqq C(1+\gamma \mu)\|u\|_{\mathscr{H}_{k-1,-r}}
\end{aligned}
$$

In the same manner we have ii).

Lemma 3.4. For $u \in \mathscr{H}_{k-2,-r}$

$$
\left\|\left(H_{\nu} \Lambda^{k-1} b(t)-b(t) H_{\nu} \Lambda^{k-1}\right) u\right\|_{\mathscr{H}_{-r}} \leqq C \mu\|u\|_{\mathscr{H}_{k-2,-\gamma}}(\nu=\mu, \delta) .
$$

Proof. Since we have $\stackrel{\circ}{H}_{\nu}=\sum_{l, m} a_{l m}^{\nu}(t, y) Y_{l m}(\gamma, \tau, \eta)$ and

$$
\Lambda=\sum_{i=0}^{n-1}(\tau, \eta)_{i} R_{i}(\gamma, \tau, \eta)+\gamma R_{r}(\gamma, \tau, \eta),
$$

on account of (**)-ii)' Lemma B give the desired result.

Lemma 3.5. For $u \in \mathscr{H}_{k-1,-r}$ the following estimates hold

i) $\quad\left\|\left\{b(t) H_{\delta} \Lambda-\Lambda b(t) H_{\delta}\right\} \Lambda^{k-1} u\right\|_{\mathscr{H}_{-r}} \leqq C\left((1+\gamma \mu)\|b(t) u\|_{\mathscr{H}_{k-1,-r}}\right.$

$$
\left.+\delta\|u\|_{\mathscr{H}_{k-1,-r}}\right) \text {, }
$$

ii) $\quad\left\|\left\{H_{\delta}^{*} \Lambda-\Lambda H_{\delta}^{*}\right\} b(t) \Lambda^{k-1} u\right\|_{\mathscr{H}_{-\gamma}} \leqq C\left((1+\gamma \mu)\|b(t) u\|_{\mathscr{H}_{k-1,-\gamma}}\right.$

$$
\left.+\delta\|u\|_{\mathscr{H}_{k-1,-r}}\right) \text {. }
$$

Proof. i) It is sufficient to prove i) in case $k=1$. We have

$$
\begin{aligned}
& \left(\Lambda b(t) H_{\delta}-b(t) H_{\delta} \Lambda\right) u=\sum_{i=0}^{n-1} R_{i}\left(D_{t y}^{e_{i}} b(t) H_{\delta}\right) u \\
& \quad+\sum_{i=0}^{n-1}\left(R_{i} b(t) H_{\delta}-b(t) H_{\delta} R_{i}\right) D_{t y}^{e_{i}} u+\left(R_{\gamma} b(t) H_{\delta}-b(t) H_{\delta} R_{\gamma}\right) \gamma u \\
& \quad=I_{1}+I_{2}+I_{3} .
\end{aligned}
$$

From (**)-iii) it follows that $\left\|I_{1}\right\|_{\mathscr{H}_{-\gamma}} \leqq C \delta\|u\|_{\mathscr{H}_{-\gamma}}$. In the same way as in the proof of Lemma 3.4 we have

$$
\left\|I_{3}\right\|_{\mathscr{H}_{-\gamma}} \leqq\left\|\left(R_{\gamma} H_{\delta}-H_{\delta} R_{\tau}\right) \gamma b(t) u\right\|_{\mathscr{H}_{-\gamma}}+C \mu\|u\|_{\mathscr{H}_{-\gamma}}
$$

from $(* *)-\mathrm{ii})$

$$
\leqq C\left(\gamma \mu\|b(t) u\|_{\mathscr{H}_{-\gamma}}+\delta\|u\|_{\mathscr{H}_{-\gamma}}\right) .
$$


Also we have

$$
\begin{aligned}
\| & I_{2} \|_{\mathscr{H}_{-r}} \leqq C\left(\sum_{i=0}^{n-1}\left\|\left(R_{i} H_{\delta}-H_{\delta} R_{i}\right) D_{t y}^{e_{i}}(b(t) u)\right\|_{\mathscr{H}_{-r}}+\delta\|u\|_{\mathscr{H}_{-r}}\right) \\
\leqq & C\left\{\sum _ { i = 0 } ^ { n - 1 } \left(\left\|\left(R_{i} H_{\delta}-R_{i} \circ H_{\delta}\right) D_{t y}^{e_{i}}(b(t) u)\right\|_{\mathscr{H}_{-r}}\right.\right. \\
& \left.\left.+\left\|\left(R_{i} \circ H_{\delta}-H_{\delta} R_{i}\right) D_{t y}^{e_{i}}(b(t) u)\right\|_{\mathscr{H}_{-r}}\right)+\delta\|u\|_{\mathscr{H}_{-r}}\right\} \\
\leqq & C\left((1+\gamma \mu)\|b(t) u\|_{\mathscr{H}_{-\gamma}}+\delta\|u\|_{\mathscr{H}_{-r}}\right) \quad(\text { by }(3.4)) .
\end{aligned}
$$

ii) In the same manner as derived (3.4) we can easily check that

$$
\left.\left\|\left(R_{i} H_{\delta}^{*}-R_{i} \circ H_{\delta}^{*}\right) D_{t y}^{\delta_{i}}(b(t) u)\right\|_{\mathscr{H}_{-\gamma} \leqq C} \leqq(1+\gamma \mu)\|b(t) u\|_{\mathscr{H}_{-\gamma}}+\delta\|u\|_{\mathscr{H}_{-\gamma}}\right) .
$$

Thus ii) follows by the same argument almost identical to the preceding one.

Combining Lemma 3.4 with Lemma 3.5 imply

Lemma 3.6. For $u \in \mathscr{H}_{k-1,-r}$

$$
\begin{array}{r}
\left\|\left(\Lambda b(t) H_{\delta}-H_{\delta} \Lambda b(t)\right) \Lambda^{k-1} u\right\|_{\mathscr{H}_{-\gamma}} \leqq C\left((1+\gamma \mu)\|b(t) u\|_{\mathscr{H}_{k-1,-\tau}}\right. \\
\left.+\delta\|u\|_{\mathscr{H}_{k-1,-\gamma}}\right) .
\end{array}
$$

Let $u \in \mathscr{H}_{k+1,-r}(\mathbb{R} \times \Omega)$ with $\left.\mathrm{D}_{t}^{j} u\right|_{t=0}=0,0 \leqq j \leqq k$ for $\Omega$ in $\S 2$. We note by Lemma 14.1 in Ohya [6] we can see $b(t)^{-1} u \in \mathscr{H}_{k-1,-r}(\mathbb{R} \times \Omega)$. Then we set

$$
\begin{aligned}
& \Psi_{k}(u)=(1+\gamma \mu)\left\{\|u\|_{\mathscr{H}_{k-1,-\gamma}}^{2}(R \times \Omega)\right. \\
& \left.\quad+\sum_{j=0}^{k-1}\left\|D_{x}^{j} u\right\|_{\mathscr{H}_{k-3 / 2-j,-\gamma}}^{2(R \times \partial \Omega)}\right\} \\
& \quad+\left\|b(t)^{-i} D_{x}^{j} D_{t y}^{\nu} u\right\|_{\mathscr{H}_{-1 / 2, \gamma}}^{2} \gamma^{-1} \delta\left\{\left\|b(t)^{-i} D_{x}^{j} D_{t y}^{\nu} u\right\|_{\mathscr{H}_{0,-\gamma}}^{2}\right\}
\end{aligned}
$$

and

$$
\phi_{k}(u)=\delta \sum_{i+j+! \nu \mid \leqq k-1}\left\|b(t)^{-i} D_{x}^{j} D_{t y}^{\nu} u\right\|_{\mathscr{H}_{0,-\gamma}(\boldsymbol{R} \times \Omega)}+(1+\gamma \mu)\|u\|_{\mathscr{H}_{k-1,-\gamma}^{(R \times \Omega)}} .
$$

The following notation is introduced for our convenience. $\stackrel{k}{\sim}$ means that the difference between the right and left side terms in some equality is dominated by const. $\Psi_{k}(u) \stackrel{k}{\cong}$ means that the difference between the right hand side and left hand side terms in the equality represents a function whose $\mathscr{H}_{-r}$-norm does not exceed const. $\phi_{k}(u)$. 
Let $Q \in \mathscr{B}_{\alpha, \infty}$. Since we take $\gamma$ sufficiently large and choose $\mu$ away from 0 in $\S 4$, we may assume for any $s>0 \gamma^{-s}$ is small enough and $\mu^{-1}<c_{1}$ for some constant $c_{1}>0$.

Lemma 3.1'. For $H$ and $H_{1}$ appeared in Lemma 3.1 and $u \in \mathscr{H}_{k+1,-r}$ with $D_{t}^{j} u=0(0 \leqq j \leqq k)$ on $t=0$, there holds

$$
\left(\left(H_{1} \circ H-H_{1} H\right) \Lambda^{k} u, Q \Lambda^{k-1} u\right)_{\mathscr{C}_{-r}} \stackrel{k}{\sim} 0 .
$$

Proof. Since we may assume that it holds $\mu^{-1} \gamma^{-1} \leqq 1$ and $\mu^{-1} \delta \leqq c_{0} c_{1}$, we have by (3.1)

$$
\begin{aligned}
\mid & \left(\left(H_{1} \circ H-H_{1} H\right) \Lambda^{k} u, Q \Lambda^{k-1} u\right)_{\mathscr{H}_{-\gamma}} \mid \\
= & \left|\left((\gamma \mu)^{-1 / 2}\left(H_{1} \circ H-H_{1} H\right) \Lambda^{k} u,(\gamma \mu)^{1 / 2} Q \Lambda^{k-1} u\right)_{\mathscr{C}_{-\gamma}}\right| \\
\leqq & C\left((\gamma \mu)^{-1}(1+\gamma \mu)^{2}|| u\left\|_{\mathscr{H}_{k-1,-\gamma}}^{2}+(\gamma \mu)^{-1} \delta^{2}|| b(t)^{-1} u\right\|_{\mathscr{H}_{k-1,-\gamma}}^{2}\right. \\
& \left.+\gamma \mu\|u\|_{\mathscr{H}_{k-1,-\gamma}}^{2}\right) \\
& \leqq \\
& C\left((1+\gamma \mu)\|u\|_{\mathscr{H}_{k-1,-\gamma}}^{2}+\gamma^{-1} \delta\left\|b(t)^{-1} u\right\|_{\mathscr{H}_{k-1,-\gamma}}^{2}\right) .
\end{aligned}
$$

Therefore the lemma is proved.

As in the proof of Lemma 3.1' the following results hold from Lemma 3.2 3. 6 for $u$ defined in Lemma 3. $1^{\prime}$.

Lemma 3.2'.

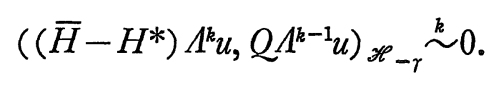

Lemma 3. $3^{\prime}$.

i) $\left(\left(\mathscr{H} \Lambda^{k}-\Lambda^{k} \mathscr{H}\right) u, Q \Lambda^{k-1} u\right)_{\mathscr{H}} \stackrel{b}{\sim} 0$.

ii) $\quad\left(\left(\mathscr{H} * \Lambda^{k}-\Lambda^{k} \mathscr{H} *\right) u, Q \Lambda^{k-1} u\right)_{\mathscr{H}_{-\tau}} \stackrel{k}{\sim} 0$.

Lemma 3. $4^{\prime}$.

$$
\begin{array}{r}
\left(\left\{\left(H_{\nu} \Lambda^{k}\right) b(t)-b(t)\left(H_{\nu} \Lambda^{k}\right)\right\} b(t)^{-1} u, Q \Lambda^{k-1} u\right)_{\mathscr{C}_{-\tau}} \stackrel{\stackrel{k}{\sim}}{\sim} 0 . \\
(\nu=\mu, \delta)
\end{array}
$$

Lemma 3.5'.

$$
\left(\left\{b(t) H_{\delta} \Lambda-\Lambda b(t) H_{\delta}\right\} \Lambda^{k-1} b(t)^{-1} u, Q \Lambda^{k-1} u\right)_{\mathscr{H}_{-\gamma}} \stackrel{k}{\sim} 0 .
$$


Lemma 3.6'.

$$
\left(\left\{\Lambda b(t) H_{\delta}-H_{\delta} \Lambda b(t)\right\} \Lambda^{k-1} b(t)^{-1} u, Q \Lambda^{k-1} u\right)_{\mathscr{H}_{-\tau}} \stackrel{k}{\sim} 0_{0}
$$

Lemma 3.7. (Gårding's inequlity) Let $\mathbb{H}=\left(H_{j k}\right)_{N \times N}$ be a hermitian matrix of order $N$ whose elements $H_{j k}$ are singular integral operators with $\sigma\left(H_{j k}\right)$ satisfying (**). Assume that for a positive constant $\delta_{1} \sigma\left(\mathbb{H}_{r}\right) \geqq \delta_{1} I$ where $\boldsymbol{H}_{r}=\left(H_{j k, r}\right)_{N \times N}$ and $H_{j k, r}$ is a regular part of $H_{j k}$. Then there exist positive constants $\delta_{1}^{\prime}$ and $C$ such that for $u={ }^{t}\left[u_{1}, \ldots, u_{N}\right] \in \mathscr{H}_{1,-r}^{N}\left(\mathbb{R}^{n}\right)$

$$
\operatorname{Re}(\boldsymbol{H} \Lambda u, \Lambda u)_{\mathscr{H}_{-r}} \geqq \delta_{1}^{\prime}\|\Lambda u\|_{\mathscr{H}_{-r}}^{2}-C\|u\|_{\mathscr{H}_{-r}}^{2}
$$

Proof. Taking account of $(* *)-\mathrm{i})$, we obtain

$$
\operatorname{Re}(\boldsymbol{H} \Lambda u, \Lambda u)_{\mathscr{H}_{-r}}=\operatorname{Re}\left(\boldsymbol{H}_{r} \Lambda u, \Lambda u\right)_{\mathscr{H}_{-r}}+\operatorname{Re}\left(\left(\mathbb{H}_{\mu}+\mathbb{H}_{\delta}\right) \Lambda u_{9} \Lambda u\right)_{\mathscr{H}_{-\gamma^{\circ}}}
$$

Since taking $\mu$ so small that $0<\mu \ll \delta_{1} \operatorname{Re}\left(\left(\mathbb{H}_{\mu}+\mathbb{H}_{\delta}\right) \Lambda u, \Lambda u\right)_{\mathscr{H}_{-\gamma}}$ is a harmless term for our estimate, we have the desired estimate by using Gårding's inequality.

Remark 3. We consider a special case where $H_{\delta} \equiv 0$ in (**). Then the condition $\delta \geqq \mu$ in (**) and (**)-iii) can be removed and we note still the same arguments as in the above go well for $\delta=0$ and $0<\mu$, too. Thus we see that all the results in this section hold for $\delta=0$ in this case.

Remark 4. Even if in Lemma 3. $1^{\prime} \sim 3.6^{\prime}$ we replace $Q \Lambda^{k-1} u$ by $\gamma^{-1} Q \Lambda^{k-1} b(t)^{-1} u$, it is seen that these lemmas remain valid. In fact, for instance, under the same hypotheses of Lemma 3.1' we consider $\left(\left(H_{1} \circ H-H_{1} H\right) \Lambda^{k} u, \gamma^{-1} Q \Lambda^{k-1} b(t)^{-1} u\right)_{\mathscr{H}_{-\gamma}}=A$. Then, rewriting

$$
A=\left((\gamma \mu)^{-1 / 2}\left(H_{1} \circ H-H_{1} H\right) \Lambda^{k} u,\left(\gamma^{-1} \mu\right)^{1 / 2} Q \Lambda^{k-1} b(t)^{-1} u\right)_{\mathscr{H}_{-\gamma}},
$$

as in the proof of Lemma $3.1^{\prime}$ we have $|A| \leqq C \Psi_{k}(u)$ 。

\section{§4. Energy Inequality}

Now we shall derive the energy estimate (1.5) and subsequently the estimate (0.7). In this section we write $s$ defined in (1.1) by $t$ again for convenience. 
We proceed the following argument according to [9]. Let $X=(t, x, y ; \tau, \eta) \in \boldsymbol{R}^{n+1} \times \boldsymbol{C}^{1} \times \boldsymbol{R}^{n-1}$ with $\operatorname{Im} \tau \leqq 0$. For a fixed $X=X_{0}$, we write $\left\{\xi_{1}^{0}, \ldots, \xi_{h}^{0}\right\}$ for the real roots of the equation $\tilde{P}_{m \xi}\left(X_{0}, \xi\right)=0$ where $\xi_{i}^{0} \neq \xi_{j}^{0}(i \neq j)$ and each $\xi_{j}^{0}$ is an $m_{j}$-tiple root. Let $X$ move aroud within a sufficiently small neighborhood $U\left(X_{0}\right)$ of $X=X_{0}$. Let

$$
H_{j}(X, \xi)=\prod_{k=1}^{m_{j}}\left(\xi-\xi_{j k}(X)\right)
$$

where $\left\{\xi_{j k}(X)\right\}_{k=1, \ldots, m_{j}}$ denote the set of roots $\xi$ of $\tilde{P}_{m \varepsilon}(x, \xi)=0$ satisfying $\xi=\xi_{j}^{0}$ at $X=X_{0}$. Let us also consider the roots $\xi$ of $\tilde{P}_{m \varepsilon}(X, \xi)=0$ in a neighborhood $U\left(X_{0}\right)$ that are complex at $X=X_{0}$ and satisfy $\operatorname{Im} \xi>0$, and call them $\left\{\xi_{1}^{+}(X), \ldots, \xi_{m_{0}^{+}}^{+}(X)\right\}$. Similarly for $\operatorname{Im} \xi<0$ we may define the set $\left\{\xi_{1}^{-}(X), \ldots, \xi_{m_{0}^{-}}^{-}(X)\right\}$. Set

$$
E_{ \pm}(X, \xi)=\prod_{j=1}^{m_{0}^{ \pm}}\left(\xi-\xi_{j}^{ \pm}(X)\right) .
$$

Therefore $\tilde{P}_{m \varepsilon}(X, \xi)$ is decomposed into products of smooth functions as

$$
\tilde{P}_{m \varepsilon}(X, \xi)=\prod_{j=1}^{h} H_{j}(X, \xi) E_{+}(X, \xi) E_{-}(X, \xi)
$$

in $U\left(X_{0}\right)$.

Consider the following polynomials of degree $m-1$ :

$$
\begin{array}{ll}
P_{j k}(X, \xi)=\left(\xi-\xi_{j}^{0}\right)^{k} \prod_{l \neq j} H_{l}(X, \xi) E_{+}(X, \xi) E_{-}(X, \xi)_{k=0, \ldots, m_{j}-1}^{j=1, \ldots, k}, \\
Q_{k}^{+}(X, \xi)=\xi^{k} \prod_{l} H_{l}(X, \xi) E_{-}(X, \xi) & \left(k=0, \ldots, m_{0}^{+}-1\right), \\
Q_{k}^{-}(X, \xi)=\xi^{k} \prod_{l} H_{l}(X, \xi) E_{+}(X, \xi) & \left(k=0, \ldots, m_{0}^{-}-1\right) .
\end{array}
$$

If $m_{j}$ is even, then $m_{j}^{+}=m_{j} / 2$. if $m_{j}$ is odd, then $m_{j}^{+}=\left(m_{j}+\varepsilon_{j}\right) / 2$ where $\varepsilon_{j}=\operatorname{sign}\left((\partial / \partial \tau) h_{j m_{j}}\right)\left(X_{0}\right)$.

Now we use the following notations: $L_{B}(X)=$ linear combinations of $\left\{B_{10}(X, \xi), \ldots, B_{m_{+} 0}(X, \xi)\right\}, L=\{$ the polynomials of degree $\mathrm{m}-1$ in

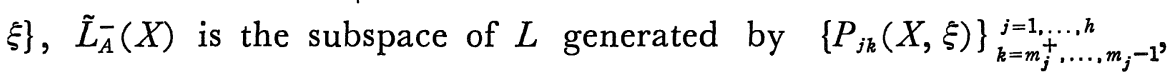
$\left\{Q_{\bar{k}}^{-}(X, \xi)\right\}_{k=0, \ldots, m_{0}^{-}-1^{\circ}}$

Proposition 1. (Sakamoto [9] PP. 142) Let $X_{0} \in\{\operatorname{Im} \tau \leqq 0,(t, x, y, \eta)$ $\left.\in \boldsymbol{R}^{2 n}\right\}$. If $R\left(X_{0}\right) \neq 0$, then $L=L_{B}(X) \oplus \tilde{L}_{\bar{A}}^{-}(X)$ in $U\left(X_{0}\right) \cap\{\operatorname{Im} \tau \leqq 0$, $\left.(t, x, y, \eta) \in \boldsymbol{R}^{2 n}\right\}$. 
In this section we write $(f, g)_{\mathscr{H}_{-\gamma}}=(f, g)_{\mathscr{H}_{0,-\tau}\left(R \times R_{+}^{n}\right)}$ and $\langle f, g\rangle_{\mathscr{H}_{-\gamma}}=\langle f, g\rangle_{\mathscr{H}_{0,-\gamma}\left(R \times R^{n-1}\right)}$.

Lemma 4.1. For $0<\alpha<1$ assume $a(t) \in C^{0}\left(\boldsymbol{R}_{+}\right)$and $t^{-\alpha}\left(t D_{t}\right)^{j} a(t)$ $\in C^{0}\left(\boldsymbol{R}_{+}\right)$for $j=1,2$ and $a(t)$ is constant for $t \geqq T_{0}$. Then there exists a constant $\beta$ with $0<\beta<1$ such that for $\varepsilon>0$

$$
\left(b(t) D_{t}\right)^{j} a\left(t_{\varepsilon}^{1 / \kappa}\right) \in \mathscr{B}_{\beta}(\boldsymbol{R}) \quad(j=0,1) \text { 。 }
$$

Proof. We take $\beta$ a irreducible fraction whose denominator is odd and $0<\beta<1$. Then we have

$$
\begin{aligned}
& \left(\partial / \partial t^{\beta}\right) a\left(t_{\varepsilon}^{1 / \kappa}\right)=\left(\partial t / \partial t^{\beta}\right)\left(\partial t_{\varepsilon} / \partial t\right) \partial / \partial t_{\varepsilon} a\left(t_{\varepsilon}^{1 / \kappa}\right) \\
& \quad=\beta^{-1} t^{1-\beta}\left(t_{\varepsilon}^{-1} t\right)\left(\kappa^{-1} t_{\varepsilon}^{1 / \kappa-1}\right) t_{\varepsilon}^{-(1-\alpha) / \kappa}\left(t_{\varepsilon}^{(1-\alpha) / \kappa} a^{\prime}\left(t_{\varepsilon}^{1 / \kappa}\right)\right) .
\end{aligned}
$$

If $\beta$ is chosen so that $-\beta+\alpha / \kappa>0$, then we have

$$
\left|\left(\partial / \partial t^{\beta}\right) a\left(t_{\varepsilon}^{1 / \kappa}\right)\right|<\infty \text { 。 }
$$

Thus we have by the mean value theorem for $t_{1}, t_{2} \in \mathbb{R}$

$$
\left|a\left(t_{1 \varepsilon}{ }^{1 / \kappa}\right)-a\left(t_{2 \varepsilon}{ }^{1 / \kappa}\right)\right| \leqq C\left|t_{1}^{\beta}-t_{2}^{\beta}\right|_{\text {。 }}
$$

Since $\left|t_{1}^{\beta}-t_{2}{ }^{\beta}\right| \leqq C\left|t_{1}-t_{2}\right|^{\beta}$, the proof of this lemma for $j=0$ is clear. Next since from the assumption it follows that there holds $t D_{t} a(t)$ $\in \mathscr{B}^{0}\left(\boldsymbol{R}_{+}\right)$and $t^{-\alpha}\left(t D_{t}\right)^{2} a(t) \in \mathscr{B}^{0}\left(\boldsymbol{R}_{+}\right)$, we can prove in the same way the case for $j=1$.

Lemma 4.2. For $0<\alpha<1$ assume that $a(t, y)$ is constant in $\{(t, y)$ $\left.\in \mathbb{R}_{+}^{n}||(t, y) \mid \geqq T_{0}\right\}$ and $t^{-\alpha} D_{y}^{\mu}\left(t D_{t}\right)^{j} a(t, y) \in C^{0}\left(\mathbb{R}_{+}^{n}\right)$ for $j \geqq 1, j+|\mu| \leqq 2$ and $D_{y}^{\mu} a(t, y) \in C^{0}\left(\boldsymbol{R}_{+}^{n}\right)$ for $|\mu| \leqq 2$. Then there exists a constant $\beta$ such that $b(t) a\left(t_{\varepsilon}^{1 / \kappa}, y\right) \in \mathscr{B}_{1+\beta}$.

Proof. From Lemma 4.1 it follows that for $\left(t_{i}, y_{i}\right) \in \mathbb{R}^{n}(\mathrm{i}=1,2)$

$$
\begin{aligned}
& \left|\left(D_{t}\left(b(t) \tilde{a}_{\varepsilon}\right)\right)\left(t_{1}, y_{1}\right)-\left(D_{t}\left(b(t) \tilde{a}_{\varepsilon}\right)\right)\left(t_{2}, y_{2}\right)\right| \\
& \quad \leqq C\left\{\left|t_{1}-t_{2}\right|^{2 \beta}+\sum_{i=1}^{n-1}\left|y_{1 i}-y_{2 i}\right|^{2 \beta}\right\}^{1 / 2} \\
& \quad \leqq 2 C\left\{\left|t_{1}-t_{2}\right|^{2}+\sum_{i=1}^{n-1}\left|y_{1 i}-y_{2 i}\right|^{2}\right\}^{\beta / 2} 。
\end{aligned}
$$

Since $\left|D_{y}^{\mu}\left(b(t) a\left(t_{\varepsilon}^{1 / \kappa}, y\right)\right)\right|_{\mathscr{B}_{1}}<\infty$ for $|\mu|=1$, we have by the interpolation theorem $D_{y}^{\mu}\left(b(t) a\left(t_{\varepsilon}^{1 / \kappa}, y\right)\right) \in \mathscr{B}_{\beta}$. Since we see immediately $b(t) a\left(t_{\varepsilon}^{1 / \kappa}, y\right)$ $\in \mathscr{B}_{1}$, the lemma is proved. 
We set $X^{\prime \prime}=(t, x, y ; \gamma, \sigma, \eta) \in \boldsymbol{R}^{n+1} \times \boldsymbol{R}_{+} \times \boldsymbol{R}^{n}$. Let $U$ be a neighborhood of $X_{0}^{\prime \prime}=\left(t_{0}, x_{0}, y_{0} ; \gamma_{0}, \sigma_{0}, \eta_{0}\right)$ in $R^{n+1} \times L^{+}$. Let symbol $a\left(X^{\prime \prime}\right)$ be difined over $U$ and be homogeneous of degree $m$ in $(\gamma, \sigma, \eta) \in \boldsymbol{R}_{+} \times \boldsymbol{R}^{n}$ and let $\phi$ be the regular symbol of degree 0 such that $\phi=1$ on some $U_{0}(\Subset U)$ and its support is contained in $U$. Define

$$
\check{a}\left(X^{\prime \prime}\right)=a\left(X^{\prime \prime}\right) \phi\left(X^{\prime \prime}\right)+\left(1-\phi\left(X^{\prime \prime}\right)\right) \Lambda^{m}(\gamma, \sigma, \eta) a\left(X_{0}^{\prime \prime}\right) .
$$

Then we say that $\check{a}\left(X^{\prime \prime}\right)$ is a standard extension of the local symbol $a\left(X^{\prime \prime}\right)$. Let $\psi(t)$ be a smooth function in $t$ such that $\operatorname{supp} \psi=\{|t| \leqq C\}$ and $\phi(t)=1$ in $\{|t| \leqq C / 2\}$ for a constant $G>0$.

Now restrict our attension on the symbol $A_{\varepsilon}\left(X^{\prime \prime}\right)=\check{\tilde{a}}_{\varepsilon}\left(X^{\prime \prime}\right)$ defined for a local symbol $a\left(X^{\prime \prime}\right) \in \mathscr{B}_{\rho, \infty}(U)(\rho=2,3, \ldots)$ which is constant in $U \cap\left\{(t, x, y) \in \boldsymbol{R} \times \boldsymbol{R}^{n}||(t, x, y) \mid \geqq T_{0}\right\} \times L^{+}$. Then for a constant $0<\mu<1$ we express $A_{\varepsilon}\left(X^{\prime \prime}\right)$ as

$$
A_{\varepsilon}\left(X^{\prime \prime}\right)=a_{r}\left(X^{\prime \prime}\right)+a_{\mu}\left(X^{\prime \prime}\right)+\alpha\left(X^{\prime \prime}\right),
$$

where

$$
\begin{aligned}
& a_{r}\left(X^{\prime \prime}\right)=\tilde{a}_{\varepsilon}\left(\hat{X}_{0}^{\prime \prime}\right) \phi\left(X^{\prime \prime}\right)+\tilde{a}_{\varepsilon}\left(X_{0}^{\prime \prime}\right)\left(1-\phi\left(X^{\prime \prime}\right)\right), \\
& a_{\mu}\left(X^{\prime \prime}\right)=\left(\tilde{a}_{\varepsilon}\left(X^{\prime \prime}\right)-\tilde{a}_{\varepsilon}\left(\hat{X}_{0}^{\prime \prime}\right)\right) \phi\left(X^{\prime \prime}\right)(1-\phi(t)), \\
& \alpha\left(X^{\prime \prime}\right)=\left(\tilde{a}_{\varepsilon}\left(X^{\prime \prime}\right)-\tilde{a}_{\varepsilon}\left(\hat{X}_{0}^{\prime \prime}\right)\right) \phi\left(X^{\prime \prime}\right) \phi(t)
\end{aligned}
$$

with $\hat{X}_{0}^{\prime \prime}=\left(t_{0}, x_{0}, y_{0} ; \gamma, \sigma, \eta\right)$. Thus if we take $U\left(X_{0}^{\prime \prime}\right)$ small enough, by using Lemma 4.2 for an integer $h \geqq 0$ there exist positive constants $\delta$ and $\beta<1$ such that it holds $\mu \leqq \delta$ and for $\Delta=R_{+}^{n+1} \times L^{+}$,

$$
(* * *)\left\{\begin{array}{l}
\left|a_{\mu}\right|_{\mathscr{B}_{0, h}(\Delta)},|\alpha|_{\mathscr{B}_{0, h}(\Delta)}<\mu, \\
\sup _{x \in R_{+}}|b(t) \alpha|_{\mathscr{B}_{1+\beta, h}},\left|D_{y}^{\nu} D_{x}^{i}\left(b(t) D_{t}\right)^{j} \alpha\right|_{\mathscr{B}_{0, h}(\Delta)}<\delta, \text { for } i+j+|\nu| \leqq \rho \\
a_{r}\left(X^{\prime \prime}\right) \text { is a regular symbol, } a_{\mu}\left(X^{\prime \prime}\right) \in \mathscr{B}_{\rho, \infty}(\Delta) \text { and } a_{\mu} \text { and } a_{\delta} \text { are } \\
\text { regualr in }\left\{(t, x, y) \in R^{n+1}|| t \mid \geqq T_{0}^{\kappa}\right\} \times L^{+} .
\end{array}\right.
$$

Let $S_{\alpha}=\left\{a\left(X^{\prime \prime}\right) \mid a\left(X^{\prime \prime}\right)\right.$ satisfies the properties of $\alpha$ in (***)\} and denote a symbol class defined by replacing $\alpha$ by $a_{\mu}$ in the definition of $S_{\alpha}$ by $S_{\mu}$. Then we remark that $S_{\alpha} \supset S_{\mu}$ for sufficiently large $\delta$. Now we denote $\alpha\left(X^{\prime \prime}\right)$ by $a_{\delta}\left(X^{\prime \prime}\right)$. In the following argument we take $h$ appropriately large.

If a symbol $P\left(X^{\prime \prime}\right)=p_{r}\left(X^{\prime \prime}\right)+p_{\mu}\left(X^{\prime \prime}\right)+p_{\delta}\left(X^{\prime \prime}\right)$ satisfies (***), we say $P\left(X^{\prime \prime}\right)$ is a symbol of the type $A\left(X^{\prime \prime}\right)$. Then we can observe that symbols of the type $A\left(X^{\prime \prime}\right)$ satisfy (**) and for any fixed $\varepsilon, \gamma \in \boldsymbol{R}_{+}$ 
Lemma A and B hold for singular integral operators with these symbols. Therefore all the result in $\S 3$ hold for these operators. We use sometimes the following notation in this section. If $P\left(X^{\prime \prime}, \xi\right)$ $=\sum_{i=0}^{k} p_{i}\left(X^{\prime \prime}\right) \xi^{k-i} \Lambda^{i}(\gamma, \sigma, \eta)$ where $\xi \in \mathbb{R}_{k}$ and $p_{i}\left(X^{\prime \prime}\right)$ is a symbol of the type $A\left(X^{\prime \prime}\right)$, we write $P_{\nu}\left(X^{\prime \prime}, \xi\right)=\sum_{i=0}^{k} p_{i \nu}\left(X^{\prime \prime}\right) \xi^{k-i} \Lambda^{i} \quad(\nu=r, \mu$ and $\delta)$.

From now on for any local symbol our concern is only the operator with its standard extension. Therefore we write $a\left(X^{\prime \prime}\right)$ for $\check{a}\left(X^{\prime \prime}\right)$ simply。

Lemma 4.3. Let $p\left(X^{\prime \prime}\right)$ and $q\left(X^{\prime \prime}\right)$ be symbols of the tvpe $A\left(X^{\prime \prime}\right)$. Then for $k \in N$ and $u \in \mathscr{H}_{k+1,-r}$ with $\left.D_{t}^{j} u\right|_{t=0}=0(0 \leqq j \leqq k)$, there holds

$$
\left(p \Lambda^{k} u, q \Lambda^{k-1} u\right)_{\mathscr{H}_{-r}} \stackrel{k}{\sim}\left(p \Lambda^{k-1} u, q \Lambda^{k} u\right)_{\mathscr{H}_{-r^{\circ}}}
$$

Proof. It suffices to prove (4.5) for $k=1$. We begin with the proof of (4.5) for the case where $p=p_{\delta}$ and $q=q_{\delta \circ}$. We denote $b(t)$ by $t$ for simplicity. By using Lemma $3.6^{\prime}$

$$
\begin{aligned}
& \left(p_{\delta} \Lambda u, q_{\delta} u\right)_{\mathscr{H}_{-r}} \\
& \quad \stackrel{1}{\sim}\left(\Lambda t p_{\delta} t^{-1} u, q_{\delta} u\right)_{\mathscr{H}_{-r}}=\left(p_{\delta} u, \Lambda t q_{\delta} t^{-1} u\right)_{\mathscr{H}_{-r}} \\
& \quad+\left(\left[p_{\delta}, t\right] t^{-1} u, \Lambda t q_{\delta} t^{-1} u\right)_{\mathscr{H}_{-r}}+\left(\Lambda t p_{\delta} t^{-1} u,\left[q_{\delta}, t\right] t^{-1} u\right)_{\mathscr{H}_{-\gamma^{\circ}}}
\end{aligned}
$$

By the representation $\stackrel{\circ}{\delta}_{\delta}\left(X^{\prime \prime}\right)=\sum_{l, m} a_{l m}^{\delta} Y_{l m}$ given in (2.2) we have

$$
\Lambda\left[p_{\delta}, t\right] \cdot=\sum_{l, m}\left\{\sum_{i=0}^{n-1} R_{i}\left(D_{t y}^{e_{i}} a_{l m}\right)\left[Y_{l m}, t\right] \cdot+A_{l m} \cdot\right\}
$$

where $A_{l m} \cdot=\sum_{i=0}^{n-1} R_{i}\left(a_{l m}^{\delta} D_{t y}^{e_{i}}\left[Y_{l m}, t\right] \bullet\right)+\gamma R_{\gamma}\left(a_{l m}^{\delta}\left[Y_{l m}, t\right] \bullet\right)$. We observe

the second term of the right hand side of (4.6)

$$
\begin{aligned}
= & \left(t \Lambda\left[p_{\delta}, t\right] t^{-1} u, q_{\delta} t^{-1} u\right)_{\mathscr{H}_{-\gamma}} \\
= & \left(\sum_{l, m}\left\{t \sum_{i=0}^{n-1} R_{i}\left(D_{t y}^{e_{i}} a_{l m}\right)\left[Y_{l m}, t\right] t^{-1} u+t A_{l m} t^{-1} u\right\}, q_{\delta} t^{-1} u\right)_{\mathscr{H}_{-\gamma}} \\
& \stackrel{1}{\sim} \sum_{l, m}\left(\gamma^{-1 / 2} t A_{l m} t^{-1} u, \gamma^{1 / 2} q_{\delta} t^{-1} u\right)_{\mathscr{H}_{-\gamma}}(\text { by Lemma A-ii) }) \\
& \stackrel{1}{\sim} \sum_{l, m}\left(\gamma^{-1 / 2} A_{l m} t^{-1} u, \gamma^{1 / 2} q_{\delta} u\right)_{\mathscr{H}_{-\gamma}} \text { (by Lemma 3.4) } \\
& \stackrel{1}{\sim} 0 \text { (by Lemma A-ii)). }
\end{aligned}
$$

In the same manner we have the last term of the right hand side of (4. 6$) \stackrel{1}{\sim} 0$. Hence we obtain 


$$
\begin{aligned}
& \left(p_{\delta} \Lambda u, q_{\delta} u\right)_{\mathscr{H}_{-r}} \stackrel{1}{\sim}\left(p_{\delta} u, \Lambda t q_{\delta} t^{-1} u\right)_{\mathscr{H}_{-r}} \\
& \left.\stackrel{1}{\sim}\left(p_{\delta} u, q_{\delta} \Lambda u\right)_{\mathscr{H}_{-\gamma}} \text { (by using Lemma } 3.6^{\prime}\right) .
\end{aligned}
$$

Since the proof of (4.5) for other cases are more easy, we omit it.

Lemma 4.4. For non-negative integer $m$ let $H\left(X^{\prime \prime}\right)$ and $H_{1}\left(X^{\prime \prime}\right)$ be symbols of the type $A\left(X^{\prime \prime}\right)$ with $\rho \geqq \max (m, 2)$ where $\rho$ is appeared in (***). For an integer $l \geqq 0$ and $u \in \mathscr{H}_{m+l+1,-r}$ with $\left.D_{t}^{j} u\right|_{t=0}=0(0 \leqq j \leqq m+l)$,

$$
\left\|\left(H_{1} \Lambda^{m} b(t)^{m} H_{\delta} \Lambda^{l} b(t)^{-m}-H_{1} \Lambda^{m} \circ H_{\delta} \Lambda^{l}\right) u\right\|_{\mathscr{H}_{-r}} \leqq C \phi_{l+m}(u) .
$$

Proof. We denote $b(t)$ by $t$ for simplicity. We carry out the proof by induction on $m$. (4.7) holds for $m=0$. In fact, by using Lemma 3.1, we can easily prove it. Also (4.7) for $m=1$ is valid. In fact, from Lemma 3.6 it follows that

$$
H_{1} \Lambda t H_{\delta} \Lambda^{l} t^{-1} u \stackrel{l+1}{\cong} H_{1} H_{\delta} \Lambda t \Lambda^{l} t^{-1} u \stackrel{l+1}{\cong} H_{1} H_{\delta} \Lambda^{l+1} u .
$$

Since $H_{1} \Lambda \circ H_{\delta} \Lambda^{l}=H_{1} \circ H_{\delta} \Lambda^{l+1}$, by using Lemma 3.1 we arrive at (4.7) for $m=1$.

For an integer $m_{0} \geqq 2$ suppose (4.7) is valid for $m \leqq m_{0}$ and any integer $l \geqq 0$. Then we have

$$
\begin{aligned}
& H_{1} \Lambda^{m_{0}+1} t^{m_{0}+1} H_{\delta} \Lambda^{l} t^{-\left(m_{0}+1\right)} u \\
& \quad=H_{1} \Lambda^{m_{0}-1}\left(\gamma^{2}+\sum_{i=1}^{n-1} D_{y_{i}}^{2}+D_{t}^{2}\right) t^{m_{0}+1} H_{\delta} \Lambda^{l}\left(t^{-\left(m_{0}+1\right)} u\right)
\end{aligned}
$$

by Leibniz's formula

$$
\begin{aligned}
= & H_{1} \Lambda^{m_{0}-1} t^{m_{0}-1} H_{\delta} \Lambda^{l+2}\left(t^{-\left(m_{0}-1\right)} u\right) \\
& +H_{1} \Lambda^{m_{0}-1} t^{m_{0}-1}\left[t^{2}, H_{\delta} \Lambda^{l+2}\right]\left(t^{-\left(m_{0}+1\right)} u\right) \\
& +\sum_{i=1}^{n-1} \sum_{j=0}^{1}\left(\begin{array}{l}
2 \\
j
\end{array}\right) H_{1} \Lambda^{m_{0}-1} t^{m_{0}+1}\left(D_{y_{i}}^{2-j} H_{\delta}\right) \Lambda^{l} D_{y_{i}}^{j}\left(t^{-\left(m_{0}+1\right)} u\right) \\
& +H_{1} \Lambda^{m_{0}-1}\left\{\sum_{i=0}^{2}\left(\begin{array}{l}
2 \\
i
\end{array}\right)\left(D_{t}^{i} t^{m_{0}+1}\left(D_{t}^{2-i} H_{\delta}\right) \Lambda^{l}\left(t^{-\left(m_{0}+1\right)} u\right)\right)\right\} \\
& +H_{1} \Lambda^{m_{0}-1} t^{m_{0}} 2\left(-i\left(m_{0}+1\right) b^{\prime}(t) H_{\delta}+t D_{t} H_{\delta}\right) D_{t} \Lambda^{l}\left(t^{-\left(m_{0}+1\right)} u\right) \\
& =I_{1}+I_{2}+I_{3}+I_{4}+I_{5} .
\end{aligned}
$$

Set $2\left(-i\left(m_{0}+1\right) b^{\prime}(t) H_{\delta}+t D_{t} H_{\delta}\right) D_{t} \Lambda^{l}=H_{\delta}^{(l+1)}$. Then we have by Leibniz's formula 


$$
\left\|I_{5}\right\|_{\mathscr{H}_{-\gamma}} \leqq C \sum_{|\alpha| \leqq m_{0}-1} \sum_{j=0}^{\alpha_{0}} \sum_{i=0}^{\alpha_{0}-j}\left\|D_{t y}^{\alpha-\alpha_{0} e_{0}}\left\{t^{m_{0}-j}\left(D_{t}^{\alpha_{0}-j-i} H_{\delta}^{(l+1)}\right) D_{t}^{i} t^{-m_{0}-1} u\right\}\right\|_{\mathscr{H}_{-\gamma}}
$$

setting

$$
\sum_{j=0}^{\alpha_{0}-i} t^{\alpha_{0}-j-i}\left(D_{t}^{\alpha_{0}-j-i} H_{\delta}^{(l+1)}\right) D_{t}^{i}=\tilde{H}_{\delta}^{(l+1+i)}
$$

for $0 \leqq i \leqq \alpha_{0}$

$$
\begin{aligned}
(4.8) \leqq & C_{|\alpha| \leqq m_{0}-1} \sum_{i=0}^{\alpha_{0}}\left\{\left\|D_{y}^{\alpha^{\prime}}\left[t^{m_{0}-\alpha_{0}+i}, \tilde{H}_{\delta}^{(l+1+i)}\right] t^{-\left(m_{0}+1\right)} u\right\|_{\mathscr{H}_{-\gamma}}\right. \\
& \left.+\left\|D_{y}^{\alpha^{\prime}} \tilde{H}_{\delta}^{(l+1+i)}\left(t^{-1-\alpha_{0}+i} u\right)\right\|_{\mathscr{H}_{-\tau}}\right\}
\end{aligned}
$$

applying Lemma A-ii) to the first term of (4.8)

$$
\begin{aligned}
\leqq & C\left\{\phi_{m_{0}+l+1}(u)\right. \\
& \left.+\sum_{|\alpha| \leq m_{0}-1} \sum_{i=0}^{\alpha_{0}} \sum_{j=1}^{m_{0}-\alpha_{0}+i}\left\|D_{y}^{\alpha^{\prime}} t^{m_{0}-\alpha_{0}+j-i}\left(\left(\frac{\partial}{\partial \tau}\right)^{j} \tilde{H}_{\delta}^{(l+1+i)}\right)\left(t^{-\left(m_{0}+1\right)} u\right)\right\|_{\mathscr{H}_{-\gamma}}\right\}
\end{aligned}
$$

repeating the process from (4.8) up to here

$$
\leqq C \psi_{m_{0}+l+1}(u) \text {. }
$$

Similarly we have

$$
\left\|I_{i}\right\|_{\mathscr{H}_{-\gamma}} \leqq C \phi_{m_{0}+l+1}(u) \quad(i=3,4) .
$$

By using Lemma A-ii) we have similarly

$$
\left\|I_{2}\right\|_{\mathscr{H}_{-\gamma}} \leqq C \psi_{m_{0}+l+1}(u) \text { 。 }
$$

Therefore we get

$$
\begin{aligned}
& H_{1} \Lambda^{m_{0}+1} t^{m_{0}+1} H_{\delta} \Lambda^{l} t^{-\left(m_{0}+1\right)} u \\
& \stackrel{m_{0}+l+1}{\cong} H_{1} \Lambda^{m_{0}-1} t^{m_{0}-1} H_{\delta} \Lambda^{l+2}\left(t^{-\left(m_{0}-1\right)} u\right) .
\end{aligned}
$$

By the assumption we have (4.7) for $m=m_{0}+1$. Hence we have completed the induction.

Remark 5. In case $l \geqq 1$ by Lemma $\mathrm{A}-\mathrm{ii})$ we have

$$
\begin{aligned}
& H_{1} \Lambda^{m} t^{m+1} H_{\delta} \Lambda^{l} t^{-(m+1)} u \\
& \stackrel{m+l}{=} H_{1} \Lambda^{m} t^{m}\left(H_{\delta} \Lambda^{l} t^{-m}-i b^{\prime}(t)\left(\frac{\partial}{\partial \tau} H_{\delta} \Lambda^{l}\right) t^{-(m+1)}\right) u \\
& \stackrel{m+l}{\cong} H_{1} \Lambda^{m} \circ H_{\delta} \Lambda^{l} u \text { (by using Lemma 4.4)。 }
\end{aligned}
$$


Lemma 4.4'. Under the same assumption of Lemma 4.4 without imposing $\left.D_{t}^{j} u\right|_{t=0}=0(0 \leqq j \leqq m+l)$ on $u$ there holds

$$
\left\|H_{1} \Lambda^{m} H_{\mu} \Lambda^{l} u-H_{1} \Lambda^{m} \circ H_{\mu} \Lambda^{l} u\right\|_{\mathscr{H}_{-r}} \leqq C(1+\gamma \mu)\|u\|_{\mathscr{H}_{l+m-1,-r}}
$$

Proof. By Lemma 3.3. we have

$$
\begin{aligned}
& \left\|H_{1} \Lambda^{m} H_{\mu} \Lambda^{l} u-H_{1} \Lambda^{m} \circ H_{\mu} \Lambda^{l} u\right\|_{\mathscr{H}_{-\gamma}} \\
& \quad \leqq\left\|H_{1} H_{\mu} \Lambda^{m+l} u-H_{1} \circ H_{\mu} \Lambda^{m+l} u\right\|_{\mathscr{H}_{-\gamma}}+C(1+\gamma \mu)\|u\|_{\mathscr{H}_{m+l-1,-\gamma}} \\
& \quad \leqq C(1+\gamma \mu)\|u\|_{\mathscr{H}_{m+l-1,-r}}(\text { by Lemma } 3.1 \text { with } \delta=0) .
\end{aligned}
$$

\section{Proposition 2. Let}

$$
P\left(X^{\prime \prime}, \xi\right)=\xi^{m}+P_{1}\left(X^{\prime \prime}\right) \xi^{m-1}+\ldots+P_{m}\left(X^{\prime \prime}\right)
$$

with $P_{i}\left(X^{\prime \prime}\right)=p_{i}\left(X^{\prime \prime}\right) \Lambda^{i}(1 \leqq i \leqq m)$ and

$$
Q\left(X^{\prime \prime}, \xi\right)=Q_{0}\left(X^{\prime \prime}\right) \xi^{m-1}+\ldots+Q_{m-1}\left(X^{\prime \prime}\right)
$$

with $Q_{i}\left(X^{\prime \prime}\right)=q_{i}\left(X^{\prime \prime}\right) \Lambda^{i}(0 \leqq i \leqq m-1)$ where $p_{i}\left(X^{\prime \prime}\right)$ and $q_{i-1}\left(X^{\prime \prime}\right)(1 \leqq i \leqq m)$ are symbols of the type $A\left(X^{\prime \prime}\right)$ with $\rho \geqq \max (m, 2)$. For $u \in \mathscr{H}_{m+1,-\gamma}$ with $\left.D_{t}^{j} u\right|_{t=0}=0 \quad(0 \leqq j \leqq m)$

$$
\left(P u, Q^{*} u\right)_{\mathscr{H}_{-\tau}}-\left(Q u, P^{*} u\right)_{\mathscr{H}_{-r}} \stackrel{m}{\sim} i<\boldsymbol{C} U_{m}, U_{m}>_{\mathscr{H}_{-\gamma}},
$$

where $\boldsymbol{C}\left(X^{\prime \prime}, \xi\right)=\boldsymbol{Q}_{0}+P_{1} \boldsymbol{Q}_{m-1}+\ldots+P_{m} \boldsymbol{Q}_{m}$ and $U_{m}={ }^{t}\left[D_{x}^{m-1} u, \Lambda D_{x}^{m-2} u, \ldots, \Lambda^{m-1} u\right]$,

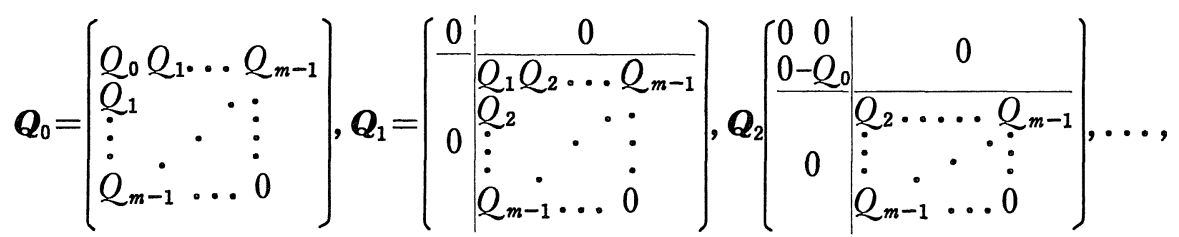

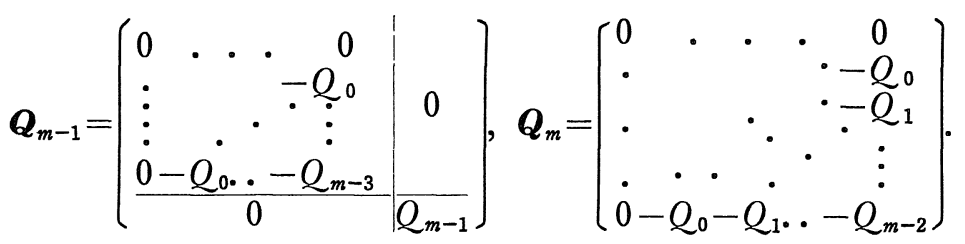

Proof. When $p_{i}\left(X^{\prime \prime}\right)=p_{i r}\left(X^{\prime \prime}\right)+p_{i \mu}\left(X^{\prime \prime}\right)$ and $q_{i-1}\left(X^{\prime \prime}\right)=q_{i-1, r}\left(X^{\prime \prime}\right)+$ $q_{i-1, \mu}\left(X^{\prime \prime}\right) \quad(1 \leqq i \leqq m)$, by the same procedure to Theorem 1 in [9] 
p. 126, we arrive at (4.10) using Lemma $3.3,3.3^{\prime}$ and Lemma $4.4^{\prime}$. Let us prove $(4,10)$ for $p_{i}\left(X^{\prime \prime}\right)=p_{i \delta}\left(X^{\prime \prime}\right)$ and $q_{i-1}\left(X^{\prime \prime}\right)=q_{i-1, \delta}\left(X^{\prime \prime}\right)$ $(1 \leqq i \leqq m)$. We prepare the following lemma.

Lemma 4.5. Let $q\left(X^{\prime \prime}\right)$ be a symbol of the type $A\left(X^{\prime \prime}\right)$. For $j+|\alpha| \leqq 1,0 \leqq i \leqq m-1$ put $F_{\nu}=D_{x}^{j} D_{t y}^{\alpha}\left(\left[\Lambda^{i} D_{x}^{m-1-i}, q_{\nu}^{*}\right] u\right)(\nu=\mu, \delta)$, then there exists a constant $G$ such that for $u \in \mathscr{H}_{m+1,-r}\left(\mathbb{R}_{+}^{n+1}\right)$ with $\left.D_{t}^{j} u\right|_{t=0}=0$ $(0 \leqq j \leqq m)$, it holds

$$
\left\|F_{\nu}\right\|_{\mathscr{H}_{-\gamma}} \leqq C \psi_{m}(u)(\nu=\mu, \delta) .
$$

Proof. When $i$ is even, on account of $\Lambda^{i}=\left(\gamma^{2}+D_{t}^{2}+\sum_{i=1}^{n-1} D_{y_{i}}^{2}\right)^{i / 2}$ we have

$$
\left\|F_{\delta}\right\|_{\mathscr{H}_{-r}} \leqq C \psi_{m}(u) .
$$

When $i$ is odd, using Leibniz's formula, we have

$$
\begin{aligned}
F_{\delta} & \stackrel{m}{\cong}\left(\Lambda q_{\delta}^{*}-q_{\delta}^{*} \Lambda\right) D_{x}^{j} D_{t y}^{\alpha} \Lambda^{i-1} D_{x}^{m-1-i} u \\
& \stackrel{m}{\cong}\left(\Lambda q_{\delta}^{*}-q_{\delta}^{*} \Lambda\right) b(t) D_{x}^{j} D_{t y}^{\alpha} \Lambda^{i-1} D_{x}^{m-1-i}\left(b(t)^{-1} u\right) \\
& \stackrel{m}{\cong} 0 \text { (by Lemma } 3.5-\mathrm{ii}) .
\end{aligned}
$$

In the same way we have using Lemma 3.3-ii)

$$
\left\|F_{\mu}\right\|_{\mathscr{H}_{-r}} \leqq C \psi_{m}(u) \text {. }
$$

We return to the proof of Proposition 2. We denote $b(t)$ by $t$ for simplicity. We have

$$
\begin{aligned}
& \left(P u, Q^{*} u\right)_{\mathscr{H}_{-r}}=\sum_{i=0}^{m} \sum_{j=0}^{m-1}\left(p_{m-i, \delta} \Lambda^{m-i} D_{x}^{i} u, D_{x}^{j} \Lambda^{m-1-j} q_{m-1-j, \delta}^{*} u\right)_{\mathscr{H}_{-r}} \\
& \stackrel{m}{\sim} \sum_{i=0}^{m} \sum_{j=0}^{m-1}\left(p_{m-i, \delta} \circ q_{m-1-j, \delta} \Lambda^{m-i} D_{x}^{i} u, D_{x}^{j} \Lambda^{m-1-j} u\right)_{\mathscr{H}_{-r}}
\end{aligned}
$$

where we write $p_{0, \delta}$ for the identity operator. In fact

i) in the case of $i=m$,

$$
\begin{aligned}
& F_{m, j}=\left(D_{x}^{m} u, D_{x}^{j} \Lambda^{m-1-j} q_{m-1-j, \delta}^{*} u\right)_{\mathscr{C}_{-\gamma}} \\
& =\left(q_{m-1-j, \delta} D_{x}^{m} u, D_{x}^{j} \Lambda^{m-1-j} u\right)_{\mathscr{H}_{-\gamma}}-<D_{x}^{m-1} u,\left[D_{x}^{j} \Lambda^{m-1-j}, q_{m-1-j, \delta}^{*}\right] u>_{\mathscr{H}_{-\gamma}} \\
& \quad+\left(D_{x}^{m-1} u, D_{x}\left[D_{x}^{j} \Lambda^{m-1-j}, q_{m-1-j, \delta}^{*}\right] u\right)_{\mathscr{H}_{-\gamma}}
\end{aligned}
$$

Since 


$$
\ll \Lambda^{1 / 2}\left[D_{x}^{j} \Lambda^{m-1-j}, q_{m-1-j, \delta}^{*}\right] u \gg_{\mathscr{H}_{-\gamma}}^{2} \leqq C\left\|\left[D_{x}^{j} \Lambda^{m-1-j}, q_{m-1-j, \delta}^{*}\right] u\right\|_{\mathscr{H}_{1,-\gamma}}^{2}
$$

by using Lemma 4.5 we have

$$
F_{m, j} \stackrel{m}{\sim}\left(q_{m-1-j, \delta} D_{x}^{m} u, D_{x}^{j} \Lambda^{m-1-j} u\right)_{\mathscr{H}_{-\gamma}} .
$$

ii) in case $i<m$, we have by Lemma 3. $6^{\prime}$

$$
\begin{aligned}
F_{i, j} & =\left(p_{m-i, \delta} \Lambda^{m-i} D_{x}^{i} u, D_{x}^{j} \Lambda^{m-1-j} q_{m-1-j, \delta}^{*} u\right)_{\mathscr{H}_{-\gamma}} \\
& \stackrel{m}{\sim}\left(\Lambda t p_{m-i, \delta} \Lambda^{m-i-1} D_{x}^{i}\left(t^{-1} u\right), D_{x}^{j} \Lambda^{m-1-j} q_{m-1-j, \delta}^{*} u\right)_{\mathscr{H}_{-r}} \\
& =\left(\Lambda t p_{m-i, \delta} \Lambda^{m-i-1} D_{x}^{i}\left(t^{-1} u\right), q_{m-1-j, \delta}^{*} D_{x}^{j} \Lambda^{m-1-j} u\right)_{\mathscr{H}_{-\gamma}} \\
& +\left(t p_{m-i, \delta} \Lambda^{m-1-i} D_{x}^{i}\left(t^{-1} u\right), \Lambda\left[D_{x}^{j} \Lambda^{m-1-j}, q_{m-1-j, \delta}^{*}\right] u\right)_{\mathscr{H}_{-\gamma}} .
\end{aligned}
$$

Since by Lemma 3.4 we have

$$
\left\|\left[t, p_{m-i, \delta}{ }^{m-1-i} D_{x}^{i}\right] t^{-1} u\right\|_{\mathscr{H}_{-\gamma}}^{2} \leqq \gamma^{-2} \mu^{2}|| t^{-1} u \|_{\mathscr{H}_{m-1,-\gamma}}^{2}
$$

taking account of Remark 4, from Lemma 4.5 it follows

the second term of (4.12)

$\stackrel{m}{\sim}\left(p_{m-i, \delta} \Lambda^{m-1-i} D_{x}^{i} u, \Lambda\left[D_{x}^{j} u, q_{m-1-j, \delta}^{*}\right] u\right)_{\mathscr{H}_{-\tau}}$

$\stackrel{m}{\sim} 0$.

We have by Lemma 4.4

$$
F_{i, j} \stackrel{m}{\sim}\left(q_{m-1-j, \delta} \circ p_{m-i, \delta} \Lambda^{m-i} D_{x}^{i} u, D_{x}^{j} \Lambda^{m-1-j} u\right)_{\mathscr{H}_{-j}}
$$

Thus we arrive at (4.11)。

In case $i \geqq j$, integrating by parts and making use of Lemma 4.3 we have

$$
\begin{aligned}
F_{i, j} \stackrel{m}{\sim} i & \left\{<p_{m-i, \delta} \circ q_{m-1-j, \delta} \Lambda^{m-i} D_{x}^{i-1} u, D_{x}^{j} \Lambda^{m-1-j} u>_{\mathscr{H}_{-\gamma}}\right. \\
& +\ldots \\
& \left.+<p_{m-i, \delta} \circ q_{m-1-j, \delta} \Lambda^{m-1-j} D_{x}^{j} u, D_{x}^{i-1} \Lambda^{m-i} u>_{\mathscr{H}_{-\gamma}}\right\} \\
& +\left(p_{m-i, \delta} \circ q_{m-1-j, \delta} \Lambda^{m-1-j} D_{x}^{j} u, D_{x}^{i} \Lambda^{m-i} u\right)_{\mathscr{H}_{-\gamma}}
\end{aligned}
$$

We have for $i \leqq m-1$ by using Lemma 4.3

$$
\text { the last term of }(4.13) \stackrel{m}{\sim}\left(p_{m-i, \delta} \circ q_{m-1-j, \delta} \Lambda^{m-j} D_{x}^{j} u, D_{x}^{i} \Lambda^{m-1-i} u\right)_{\mathscr{C}}
$$

by the reverse procedure to ii) 


$$
\stackrel{m}{\sim}\left(\Lambda t q_{m-1-j, \delta} \Lambda^{m-1-j} D_{x}^{j}\left(t^{-1} u\right), p_{m-1, \delta}^{*} D_{x}^{i} \Lambda^{m-1-i} u\right)_{\mathscr{H}_{-\gamma}}
$$

in the same procedure from $(4.6)$ to the end of the proof of Lemma 4.3

$$
\stackrel{m}{\sim}\left(q_{m-1-j, \delta} \Lambda^{m-1-j} D_{x}^{j} u, D_{x}^{i} \Lambda^{m-i} p_{m-i, \delta}^{*} u\right)_{\mathscr{H}_{-\gamma}}
$$

Taking account of $p_{0, \delta}=1$ obviously the above result remains valid for $0 \leqq i \leqq m$, too. Consequently by repeating the above procedure interchanging $i$ and $j$, we arrive at (4.10) for the case where $P\left(X^{\prime \prime}, \xi\right)=P_{\delta}\left(X^{\prime \prime}, \xi\right)+\xi^{m}$ and $Q\left(X^{\prime \prime}, \xi\right)=Q_{\delta}\left(X^{\prime \prime}, \xi\right)$.

Other cases are proved more easily. Therefore we omit it.

In the rest of this paper we denote $b(t)$ by $t$ for simplicity.

Set $\tau=\sigma-i \gamma$ for $\sigma \in \mathbb{R}$ and $\gamma \in \mathbb{R}_{+}$.

Following to Sakamoto [9] let us separate the standard extension of $H_{j}(X, \xi)$ into the two parts such that $H_{j}(X, \xi)=H_{j}^{\prime}(X, \xi)-$ $i \gamma H_{j}^{\prime \prime}(X, \xi)$ where

$$
\begin{aligned}
& H_{j}^{\prime}(X, \xi)=\left(\xi-\xi_{j}^{0} \Lambda\right)^{m_{j}}+h_{j 1}^{\prime}(X) \Lambda\left(\xi-\xi_{j}^{0} \Lambda\right)^{m_{j}-1}+\ldots+h_{j m_{j}}^{\prime}(X) \Lambda^{m_{j}}, \\
& H_{j}^{\prime \prime}(X, \xi)=h_{j 1}^{\prime \prime}(X)\left(\xi-\xi_{j}^{0} \Lambda\right)^{m_{j}-1}+\ldots+h_{j m_{j}}^{\prime \prime}(X) \Lambda^{m_{j}-1}
\end{aligned}
$$

where $h_{j i}^{\prime}(X)$ and $h_{j i}^{\prime \prime}(X)$ are real symbols for $1 \leqq i \leqq m_{j}$. Obviously these symbols have the same properties as $A_{\varepsilon}\left(X^{\prime \prime}\right)$, that is, these are symbols of the type $A\left(X^{\prime \prime}\right)$.

Lemma 4.6. For an arbitrary $0<\mu<1$, there exists a neighborhood $U^{(\mu)}$ of $X=X_{0}$ such that if $H_{j}$ is the standard extension with respect to $U^{(\mu)}$, then for $u \in \mathscr{H}_{m_{j}+1,-\gamma}$ with $\left.D_{t}^{i} u\right|_{t=0}=0\left(0 \leqq i \leqq m_{j}\right)$, there exist constants $C, C_{\mu}$ and $c_{\mu}$ such that

$$
\begin{aligned}
& c_{\mu} \gamma\|u\|_{\mathscr{H}_{m_{j}-1,-\gamma}}^{2}+\sum_{k=m_{j}^{+}}^{m_{j}-1} \ll\left(D_{x}-\xi_{j}^{0} \Lambda\right)^{k} u \gg_{\mathscr{H}_{m_{j}-1-k_{1}-\gamma}}^{2} \\
& \leqq C \mu^{2} \sum_{k=0}^{m_{j}^{+}-1} \ll\left(D_{x}-\xi_{j}^{0} \Lambda\right)^{k} u \gg_{\mathscr{H}_{m_{j}^{+}-1-k,-\gamma}^{2}}^{2}+C_{\mu}\left\{\gamma^{-1}\left\|H_{j} u\right\|_{\mathscr{H}_{-\gamma}}^{2}+\Psi_{m_{j}}(u)\right\} .
\end{aligned}
$$

Proof. Let $Q(X, \xi)$ be any regular real symbol of degree $\mathrm{m}_{j}-1$ 
such that

$$
Q(X, \xi)=Q_{0}(X)\left(\xi-\xi_{j}^{0} \Lambda\right)^{m_{j}-1}+Q_{1}(X)\left(\xi-\xi_{j}^{0} \Lambda\right)^{m_{j}-2}+\ldots+Q_{m_{j}-1}(X) .
$$

Then we observe

$$
\begin{aligned}
& \left(H_{j} u, Q^{*} u\right)_{\mathscr{H}_{-r}}-\left(Q u, H_{j}^{*} u\right)_{\mathscr{H}_{-r}} \\
& \quad=\left(H_{j}^{\prime} u, Q^{*} u\right)_{\mathscr{H}_{-r}}-\left(Q u, H_{j}^{\prime *} u\right)_{\mathscr{H}_{-r}}-i \gamma\left\{\left(H_{j}^{\prime \prime} u, Q^{*} u\right)_{\mathscr{H}_{-r}}\right. \\
& \left.\quad+\left(Q u, H_{j}^{\prime *} u\right)_{\mathscr{H}_{-r}}\right\} \\
& \quad=i I_{1}+i \gamma I_{2}
\end{aligned}
$$

By applying Proposition 2 to $I_{1}$, there exists a $m_{j} \times m_{j}$ matrix $\mathscr{H}_{j}^{\prime}$ such that for

$$
\begin{gathered}
U_{m_{j}}={ }^{t}\left[\left(D_{x}-\xi_{j}^{0} \Lambda\right){ }^{m_{j}-1} u, \Lambda\left(D_{x}-\xi_{j}^{0} \Lambda\right)^{m_{j}-2} u, \ldots, \Lambda^{m_{j}-1} u\right], \\
I_{1} \stackrel{m_{j}}{\sim}\left\langle\mathscr{H}_{j}^{\prime} U_{m_{j}}, U_{m_{j}}\right\rangle_{\mathscr{H}_{-j}}
\end{gathered}
$$

It is known in the proof of Lemma 3.4 in [9] that we can choose $Q(X, \xi)$ so that for positive constants $C$ and $C^{\prime}$ and $U={ }^{t}\left[u_{m_{j}-1}, \ldots, u_{0}\right]$

$$
{ }^{t} \bar{U} \mathscr{H}_{j}^{\prime} U \geqq C \sum_{k=m_{j}^{+}}^{m_{j}-1}\left|u_{k}\right|^{2}-C^{\prime} \mu^{2} \sum_{k=0}^{m_{j}^{+}-1}\left|u_{k}\right|^{2} .
$$

By using Lemma 3.7 we have,

$$
\begin{aligned}
I_{1} & \geqq C \sum_{k=m_{j}^{+}}^{m_{j}-1} \ll\left(D_{x}-\xi_{j}^{0} \Lambda\right)^{k} u \gg_{\mathscr{H}_{m_{j}-1-k_{1}-r}^{2}} \\
& -C^{\prime} \mu^{2^{2}} \sum_{k=0}^{m_{j}^{+}-1} \ll\left(D_{x}-\xi_{j}^{0} \Lambda\right)^{k} u \gg_{\mathscr{H}_{m_{j}-1-k_{1}-r}}^{2}-C^{\prime \prime} \Psi_{m_{j}}(u) .
\end{aligned}
$$

On the other hand, taking the neighborhood $U^{(\mu)}$ of $X=X_{0}$ sufficiently small, we have

$$
\text { (4. 17) } I_{2} \geqq c_{\mu}\|u\|_{\mathscr{H}_{m_{j}-1,-r}}^{2}-C_{\mu}\left\{\left\|\Lambda^{-1} H_{j} u\right\|_{\mathscr{H}_{-\gamma}}^{2}+\gamma^{-1} \Psi_{m_{j}}(u)\right\} \text {. }
$$

This inequality was proved for the case where $H_{j}$ is regular in the proof of Lemma 4.4 in [9]; the extension to our case is carried out in the same way taking care that $H_{j}(X, \xi)$ is not smooth on $t=0$. Since we have by Lemma $3,2^{\prime}$

$$
\begin{aligned}
\left(Q u,\left(H_{j}^{*}-H_{j}\right) u\right)_{\mathscr{H}_{-r}} \stackrel{m_{j}}{\sim}\left(Q u, \sum_{1 \leqq i}\left\{\left(\left(D_{x}-\xi_{j}^{0} \Lambda\right)^{m_{j}-i} \Lambda^{i} h_{j i}^{\prime *}\right.\right.\right. \\
\left.-h_{j i}^{* *} \Lambda^{i}\left(D_{x}-\xi_{j}^{0} \Lambda\right)^{m_{j}-i}\right) u
\end{aligned}
$$




$$
\left.\left.-i \gamma\left(\left(D_{x}-\xi_{j}^{0} \Lambda\right)^{m_{j}-i} \Lambda^{i-1} h_{j i}^{\prime \prime *}-h_{j i}^{\prime \prime *} \Lambda^{i-1}\left(D_{x}-\xi_{j}^{0} \Lambda\right)^{m_{j}-i}\right) u\right\}\right)_{\mathscr{H}_{-\gamma}}
$$

in the same way as in the proof of Lemma 4.5 we have

$$
\left(Q u,\left(H_{j}^{*}-H_{j}\right) u\right)_{\mathscr{H}_{-r}} \stackrel{m_{j}}{\sim} 0 .
$$

Therefore (4.16) (4.18) imply that the desired result holds.

Let us separate the standard extension of $E(X, \xi)=E_{+}(X, \xi) \times$ $E_{-}(X, \xi)$ into the real and imaginary parts such that

$$
E_{ \pm}(X, \xi)=E_{ \pm}^{\prime}(X, \xi)-i E_{ \pm}^{\prime \prime}(X, \xi),
$$

where

$$
\begin{aligned}
& E_{ \pm}^{\prime}(X, \xi)=\xi^{m_{0}^{ \pm}}+e_{1}^{ \pm \prime}(X) \Lambda \xi^{m_{0}^{ \pm}-1}+\ldots+e_{m_{0}}^{ \pm}(X) \Lambda^{m_{0}^{ \pm}} \\
& E_{ \pm}^{\prime \prime}(X, \xi)=e_{1}^{ \pm^{\prime \prime}}(X) \Lambda \xi^{m_{0}^{ \pm}-1}+\ldots+e_{m_{0}^{ \pm}}{ }^{\prime \prime}(X) \Lambda^{m_{0}^{ \pm}}
\end{aligned}
$$

From the arguments for $A_{\varepsilon}\left(X^{\prime \prime}\right)$ it follows immediately that $e_{i}^{ \pm \prime}(X)$ and $e_{i}^{ \pm \prime \prime}(X) \quad\left(1 \leqq i \leqq m_{0}^{ \pm}\right)$are symbols of the type $A\left(X^{\prime \prime}\right)$.

Lemma 4. 7. For $u \in \mathscr{H}_{m_{0}^{ \pm}+1,-\tau}$ with $\left.D_{t}^{j} u\right|_{t=0}=0 \quad\left(0 \leqq j \leqq m_{0}^{ \pm}\right)$it holds i) $\quad \gamma|| u \|_{\mathscr{H}_{m_{0}^{+}-1,-\gamma}^{2}} \leqq C\left\{\sum_{k=0}^{m_{0}^{+}-1}\left\langle D_{x}^{k} u\right\rangle_{\mathscr{H}_{m_{0}^{+}-1-k_{-}-\gamma}^{2}}^{2}+\gamma^{-1}|| E_{+} u \|_{\mathscr{H}_{-\gamma}}^{2}+\Psi_{m_{0}^{+}}(u)\right\}$, ii) $\quad r \mid\|u\|_{\mathscr{H}_{m_{0}^{-}-1,-\gamma}^{2}}^{2}+\sum_{k=0}^{m_{0}^{-}-1}\left\langle\left\langle D_{x}^{k} u\right\rangle_{\mathscr{H}_{m_{0}^{-}-1-k_{1}-\gamma}^{2}}^{2} \leqq C\left\{\gamma^{-1}|| E_{-} u \|_{\mathscr{H}_{-\gamma}}^{2}+\Psi_{m_{0}^{-}}(u)\right\}\right.$.

Proof. We consider

$$
\begin{aligned}
\left\|\Lambda^{-1 / 2} E_{ \pm} u\right\|_{\mathscr{H}_{-r}}^{2}=\left\|\Lambda^{-1 / 2}\left(E_{ \pm}^{\prime}-i E_{ \pm}^{\prime \prime}\right) u\right\|_{\mathscr{H}_{-}}^{2} \\
=\left\|\Lambda^{-1 / 2} E_{ \pm}^{\prime} u\right\|_{\mathscr{H}_{-\gamma}}^{2}+\left\|\Lambda^{-1 / 2} E_{ \pm}^{\prime \prime} u\right\|_{\mathscr{H}_{-}}^{2} \\
\quad+i\left\{\left(E_{ \pm}^{\prime} u, \Lambda^{-1} E_{ \pm}^{\prime \prime} u\right)_{\mathscr{H}_{-}}-\left(\Lambda^{-1} E_{ \pm}^{\prime \prime} u, E_{ \pm}^{\prime} u\right)_{\mathscr{H}_{-\gamma}}\right\}=I_{1}^{ \pm}+I_{2}^{ \pm} 。
\end{aligned}
$$

By using Lemma 3.3 and Lemma 3.6 we have

$$
\begin{aligned}
& \left\|\Lambda^{-1 / 2}\left(E_{ \pm \delta}^{\prime}+E_{ \pm \mu}^{\prime}\right) u\right\|_{\mathscr{H}_{-}}^{2} \geqq i \mid \Lambda^{1 / 2}\left(t E_{ \pm \delta}^{\prime} \Lambda^{-1} t^{-1} u+E_{ \pm \mu}^{\prime} \Lambda^{-1} u\right) \|_{\mathscr{H}_{-\gamma}}^{2}-C \Psi_{m_{0}^{ \pm}}(u) \\
& \quad \geqq \gamma\left\|\left(E_{ \pm \delta}^{\prime}+E_{ \pm \mu}^{\prime}\right) \Lambda^{-1} u\right\|_{\mathscr{H}_{-\gamma}}^{2}-C \Psi_{m_{0}^{ \pm}}(u) \quad(\text { by Lemma 3.4) } \\
& \quad \geqq-C \Psi_{m_{0}^{ \pm}}(u) .
\end{aligned}
$$

Therefore we have 


$$
\left\|\Lambda^{-1 / 2} E_{ \pm}^{\prime}(u)\right\|_{\mathscr{H}_{-\gamma}}^{2} \geqq \gamma\left\|E_{ \pm r}^{\prime} \Lambda^{-1} u\right\|_{\mathscr{H}_{-\gamma}}^{2}-C \Psi_{m_{0}^{ \pm}}(u) .
$$

Similarly we get

$$
\left\|\Lambda^{-1 / 2} E_{ \pm}^{\prime \prime} u\right\|_{\mathscr{H}_{-\gamma}}^{2} \geqq \gamma\left\|E_{ \pm r}^{\prime \prime} \Lambda^{-1} u\right\|_{\mathscr{H}_{-\gamma}}^{2}-C \Psi_{m_{0}^{ \pm}}(u) .
$$

Since in the proof of Lemma 3.5 in [9] it was established that

$$
\left\|\Lambda^{-1 / 2} E_{ \pm r}^{\prime} u\right\|_{\mathscr{H}_{-r}}^{2}+\left\|\Lambda^{-1 / 2} E_{ \pm r}^{\prime \prime} u\right\|_{\mathscr{H}_{-\gamma}}^{2} \geqq C\left\{\left\|\Lambda^{-1 / 2} u\right\|_{\mathscr{H}_{m_{0}^{ \pm},-r}}^{2}-\|u\|_{\mathscr{H}_{m_{0}^{ \pm}-1,-r}}^{2}\right\}
$$

we have

$$
I_{1}^{ \pm} \geqq C\left\{r\|u\|_{\mathscr{H}_{m_{0}^{ \pm}-1,-r}}^{2}-\Psi_{m_{0}^{ \pm}}(u)\right\} .
$$

Now we represent $\Lambda^{-1} E_{ \pm \delta}^{\prime \prime}$ as in the form

$$
\Lambda^{-1} E_{ \pm \delta}^{\prime \prime} u=\Lambda^{-1} F_{ \pm}\left(t^{-1} u\right)+G_{ \pm} \Lambda^{-1}\left(t^{-1} u\right)+E_{ \pm \delta}^{\prime \prime} \Lambda^{-1} u,
$$

where

$$
F_{ \pm}=\left[E_{ \pm \delta}^{\prime \prime} \Lambda^{-1}, \Lambda t\right] \text { and } G_{ \pm}=\left[t, E_{ \pm \delta}^{\prime \prime} \Lambda^{-1}\right] \Lambda \text {. }
$$

We note that by Lemma 3.6 and Lemma 3.4 we have

$$
\begin{aligned}
& \left\|\Lambda^{-1} F_{ \pm} t^{-1} u\right\|_{\mathscr{H}_{-\gamma}}^{2} \leqq C \gamma^{-1} \mu \Psi_{m_{0}^{ \pm}}(u) \quad \text { and } \\
& \left\|G_{ \pm} \Lambda^{-1} t^{-1} u\right\|_{\mathscr{H}_{-\gamma}}^{2} \leqq C \gamma^{-1} \mu \Psi_{m_{0}^{ \pm}}(u)
\end{aligned}
$$

Now we shall prove

$$
\begin{aligned}
\left(E_{ \pm}^{\prime} u, \Lambda^{-1} E_{ \pm}^{\prime \prime} u\right)_{\mathscr{H}_{-r}} \stackrel{m_{0}^{ \pm}}{\sim}\left(E_{ \pm}^{\prime} u, E_{ \pm}^{\prime \prime} \Lambda^{-1} u\right)_{\mathscr{H}_{-r}} \\
\quad+\left(E_{ \pm} u,\left(\Lambda^{-1} F_{ \pm}+G_{ \pm} \Lambda^{-1}\right) t^{-1} u\right)_{\mathscr{H}_{-r}}
\end{aligned}
$$

To prove this, we begin by considering

$$
\begin{aligned}
\left(E_{ \pm \delta}^{\prime \prime} u, \Lambda^{-1} F_{ \pm}\left(t^{-1} u\right)\right)_{\mathscr{H}_{-\gamma}}=\left(\gamma^{-1 / 2}\left(E_{ \pm \delta}^{\prime \prime} \Lambda^{-1}\right) \Lambda u, \gamma^{1 / 2} \Lambda^{-1} F_{ \pm}\left(t^{-1} u\right)\right)_{\mathscr{H}_{-\gamma}} \\
\quad \stackrel{m_{0}^{ \pm}}{\sim}\left(\Lambda t\left(E_{ \pm \delta}^{\prime \prime} \Lambda^{-1}\right) t^{-1} u, \Lambda^{-1} F_{ \pm} t^{-1} u\right)_{\mathscr{H}_{-\gamma}} \quad(\text { by Lemma 3.6) } \\
\quad=\left(\gamma^{1 / 2} t\left(E_{ \pm \delta}^{\prime \prime} \Lambda^{-1}\right) t^{-1} u, \gamma^{-1 / 2} F_{ \pm} t^{-1} u\right)_{\mathscr{H}_{-\gamma}} \\
\stackrel{m_{0}^{ \pm}}{\sim}\left(\gamma^{1 / 2} E_{ \pm \delta}^{\prime \prime} \Lambda^{-1} u, \gamma^{-1 / 2} F_{ \pm} t^{-1} u\right)_{\mathscr{H}_{-\gamma}}(\text { by Lemma 3.4) } \\
\quad m_{0}^{ \pm} 0 .
\end{aligned}
$$

We have by Lemma $3.3^{\prime}$ and Remark 4

$$
\left(\left(E_{ \pm r}^{\prime \prime}+E_{ \pm \mu}^{\prime \prime}\right) u, \Lambda^{-1} F_{ \pm}\left(t^{-1} u\right)\right)_{\mathscr{H}_{-\gamma}} \stackrel{m_{0}^{ \pm}}{\sim} 0
$$


Therefore we have

$$
\left(E_{ \pm}^{\prime} u, \Lambda^{-1} F_{ \pm}\left(t^{-1} u\right)\right)_{\mathscr{H}_{-r}}^{\stackrel{m_{0}^{ \pm}}{\sim}}\left(E_{ \pm} u, \Lambda^{-1} F_{ \pm}\left(t^{-1} u\right)\right)_{\mathscr{H}_{-\tau^{\circ}}}
$$

On the other hand we have by Lemma $3.6^{\prime}$ and Remark 4

$$
\left(E_{ \pm \delta}^{\prime \prime} u, G_{ \pm} \Lambda^{-1} t^{-1} u\right)_{\mathscr{H}_{-\gamma}} \stackrel{m_{0}^{ \pm}}{\sim}\left(\Lambda t\left(E_{ \pm \delta}^{\prime \prime} \Lambda^{-1}\right) t^{-1} u, G_{ \pm} \Lambda^{-1} t^{-1} u\right)_{\mathscr{H}_{-\gamma}}
$$

by the same way as used for estimating the second term of the right hand side of (4.6) in Lemma 4.3

$$
\stackrel{m_{0}^{ \pm}}{\sim} 0 \text {. }
$$

Similarly we have

$$
\begin{aligned}
& \left(\left(E_{ \pm r}^{\prime \prime}+E_{ \pm \mu}^{\prime \prime}\right) u, G_{ \pm} \Lambda^{-1} t^{-1} u\right)_{\mathscr{H}_{-r}} \\
& \quad=\left(\mu^{1 / 2}\left(E_{ \pm r}^{\prime \prime}+E_{ \pm \mu}^{\prime \prime}\right) u, \mu^{-1 / 2} G_{ \pm} \Lambda^{-1} t^{-1} u\right)_{\mathscr{H}_{-r}} \\
& \quad \stackrel{m_{0}^{ \pm}}{\sim} 0 .
\end{aligned}
$$

Therefore we obtain

$$
\left(E_{ \pm}^{\prime} u, G_{ \pm} \Lambda^{-1}\left(t^{-1} u\right)\right)_{\mathscr{H}_{-\gamma}} \stackrel{m_{0}^{ \pm}}{\sim}\left(E_{ \pm} u, G_{ \pm} \Lambda^{-1}\left(t^{-1} u\right)\right)_{\mathscr{H}_{-\tau}}
$$

Combining (4.22) with (4.23) implies (4.21). From (4.21) it follows immediately

$$
\begin{aligned}
& I_{2}^{ \pm} \stackrel{m_{0}^{ \pm}}{\sim} i\left\{\left(E_{ \pm}^{\prime} u, E_{ \pm}^{\prime \prime} \Lambda^{-1} u\right)_{\mathscr{H}_{-r}}-\left(E_{ \pm}^{\prime \prime} \Lambda^{-1} u, E_{ \pm}^{\prime} u\right)_{\mathscr{H}_{-\gamma}}\right. \\
& \left.\quad+2 \operatorname{Im}\left(E_{ \pm} u,\left(\Lambda^{-1} F_{ \pm}+G_{ \pm} \Lambda^{-1}\right) t^{-1} u\right)_{\mathscr{H}_{-\gamma}}\right\}
\end{aligned}
$$

We have

$$
\begin{aligned}
& \left|2 \operatorname{Im}\left(E_{ \pm} u,\left(\Lambda^{-1} F_{ \pm}+G_{ \pm} \Lambda^{-1}\right) t^{-1} u\right)_{\mathscr{H}_{-\gamma}}\right| \\
& \quad \leqq C\left(\gamma^{-1}|| E_{ \pm} u \|_{\mathscr{H}_{-\gamma}}^{2}+\Psi_{m_{0}^{ \pm}}(u)\right) .
\end{aligned}
$$

We denote $E_{ \pm}^{\prime \prime} \Lambda^{-1}$ by $K_{ \pm}$for simplicity. Let

$$
I_{2}^{ \pm \prime}=i\left\{\left(E_{ \pm}^{\prime} u, K_{ \pm}^{*} u\right)_{\mathscr{H}_{-r}}-\left(K_{ \pm} u, E_{ \pm}^{\prime}{ }^{*} u\right)_{\mathscr{H}_{-r}}\right\} 。
$$

From Proposition 2 it follows that there exists a $m_{0}^{ \pm} \times m_{0}^{ \pm}$matrix $\mathscr{E}_{ \pm}(X)$ such that

$$
I_{2}^{ \pm} \prime_{0}^{m_{0}^{ \pm}} \sim<\mathscr{E}_{ \pm} U_{m_{0}^{ \pm}}, U_{m_{0}^{ \pm}}>_{\mathscr{H}_{-\gamma}\left(R^{n}\right)^{\circ}}
$$


Then it is known in [9] that the following inequality holds for $|\tau|^{2}+|\eta|^{2}=1$ and $U={ }^{t}\left[u_{m_{0}^{ \pm}-1}, \ldots, u_{0}\right]$

$$
\pm^{t} \bar{U} \mathscr{E}_{ \pm}(X) U \geqq C \sum_{k=0}^{m_{0}^{ \pm}-1}\left|u_{k}\right|^{2} .
$$

From Lemma 3.7 it follows that

$(4.26)_{+} \quad I_{2}^{+\prime} \geqq-C\left\{\sum_{k=0}^{m_{0}^{+}-1}\left\langle D_{x}^{k} u\right\rangle_{\mathscr{H}_{m_{0}^{+}-1-k_{1}-r}^{2}}+\Psi_{m_{0}^{+}}(u)\right\}$,

(4. 26) - $\quad I_{2}^{-\prime} \geqq C\left\{\sum_{k=0}^{m_{0}^{-}-1} \ll D_{x}^{k} u \gg_{\mathscr{H}_{m_{0}^{-}-1-k_{1}-r}^{2}}^{2}-\Psi_{m_{0}^{-}}(u)\right\}$.

By the same argument as derived (4.18) we have

$$
\left(\left(E_{ \pm}^{\prime}{ }^{*}-E_{ \pm}^{\prime}\right) u, K_{ \pm} u\right)_{\mathscr{H}_{-r}} \stackrel{m_{0}^{ \pm}}{\sim} 0 .
$$

On the other hand

$$
\begin{aligned}
\left(E_{ \pm}^{\prime} u,\left(K_{ \pm}^{*}-K_{ \pm}\right) u\right)_{\mathscr{H}_{-r}}= & \left(E_{ \pm} u,\left(K_{ \pm}^{*}-K_{ \pm}\right) u\right)_{\mathscr{H}_{-\gamma}} \\
& -i\left(E_{ \pm}^{\prime \prime} u,\left(K_{ \pm}^{*}-K_{ \pm}\right) u\right)_{\mathscr{H}_{-\tau}}
\end{aligned}
$$

by the same way as used for deriving (4.12) and as derived (4.15) from (4.14)

$$
\stackrel{m_{0}^{ \pm}}{\sim}\left(E_{ \pm} u,\left(K_{ \pm}^{*}-K_{ \pm}\right) u\right)_{\mathscr{H}_{-\gamma}}-i\left(E_{ \pm}^{\prime \prime} \Lambda^{-1} u, \quad\left(K_{ \pm}^{*}-K_{ \pm}\right) \Lambda u\right)_{\mathscr{H}_{-r}}
$$

by the same way as derived (4.18)

$$
\stackrel{m_{0}^{ \pm}}{\sim}\left(E_{ \pm} u,\left(K_{ \pm}^{*}-K_{ \pm}\right) u\right)_{\mathscr{H}_{-\tau}} \text {. }
$$

Similarly the absolute value of (4.27) is dominated by const. $\left(\gamma^{-1}|| E_{ \pm} u \|_{\mathscr{H}}^{2}+\gamma+\Psi_{m_{0}^{ \pm}}(u)\right)$. Consequently we obtain

$$
\left|I_{2}^{ \pm}-I_{2}^{ \pm \prime}\right| \leqq C\left(\gamma^{-1}|| E_{ \pm} u \|_{\mathscr{H}_{-\gamma}}^{2}+\Psi_{m_{0}^{ \pm}}(u)\right) .
$$

Hence by (4.19), (4.24) (4.26) $)_{ \pm}$and the just obtained inequality we get the desired result.

Here and in the rest of this paper let $u$ be the solution of (1.4). We consider the symbol $\tilde{P}_{m \varepsilon}$ in a neighborhood $U\left(X_{0}\right)$ of $X=X_{0}$ as

$$
\begin{gathered}
\tilde{P}_{m \varepsilon}(X, \xi)=\prod_{j=1}^{h} H_{j}(X, \xi) E_{+}(X, \xi) E_{-}(X, \xi)=H_{1}(X, \xi) P_{10}(X, \xi)=\ldots \\
=H_{h}(X, \xi) P_{h 0}(X, \xi)=E_{+}(X, \xi) Q_{0}^{+}(X, \xi)=E_{-}(X, \xi) Q_{0}^{-}(X, \xi)
\end{gathered}
$$


and the corresponding operators with standard extension with respect to $U\left(X_{0}\right)$. Now we introduce a neighborhood of $X_{0}: U_{1}$ such that $X_{0} \in U_{1} \Subset U_{0} \Subset U$. Let $\phi_{0}$ be a regular symbol of degree 0 such that supp $\phi_{0} \subset U_{0}$ and $\phi_{0}=1$ on $U_{1}$. Then by Remark 5 and Lemma $4_{\circ} 4^{\prime}$ we have

$$
\begin{aligned}
& \tilde{P}_{m \varepsilon} \phi_{0} u \stackrel{m}{\cong} \tilde{P}_{m \varepsilon} \circ \phi_{0} u \\
& \stackrel{m}{\cong} H_{1}\left\{\left(P_{10, r}+P_{10, \mu}\right) \circ \phi_{0} u+t^{m_{1}+1} P_{10, \delta^{\circ}} \phi_{0} t^{-m_{1}-1} u\right\} \stackrel{m}{\cong} \ldots \\
& \stackrel{m}{\cong} H_{h}\left\{\left(P_{h 0, r}+P_{h 0, \mu}\right) \circ \phi_{0} u+t^{m_{1}+1} P_{h 0, \delta} \circ \phi_{0} t^{-m_{h}-1} u\right\} \\
& \stackrel{m}{\cong} E_{+}\left\{\left(Q_{0 r}^{+}+Q_{0 \mu}^{+}\right) \circ \phi_{0} u+t^{m_{0}^{+}+1} Q_{0 \delta}^{+} \circ \phi_{0} t^{-m_{0}^{+}-1} u\right\} \\
& \stackrel{m}{\cong} E_{-}\left\{\left(Q_{0 r}+Q_{0 \mu}^{-}\right) \circ \phi_{0} u+t^{m_{0}^{-+1}} Q_{0 \delta}^{\circ} \phi_{0} t^{-m_{0}^{-}-1} u\right\} .
\end{aligned}
$$

Let

(4. 28) $\quad \tilde{P}_{m \varepsilon}^{\prime}(X, \xi)=\tilde{P}_{m \varepsilon}(X, \xi)-\xi^{m}$ 。

Then it holds

$$
\left\|\Lambda^{-1} \tilde{P}_{m \varepsilon}^{\prime} u\right\|_{\mathscr{H}_{-\gamma}} \leqq C\|u\|_{\mathscr{H}_{m-1,-r}}
$$

We have by (4.28)

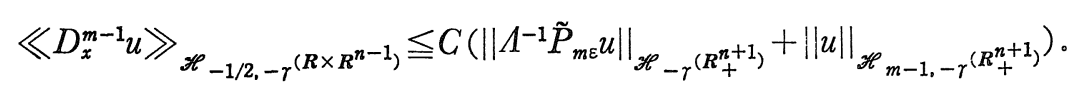

By using this estimate and the easily proved inequality for $j \in \mathbb{N}$ :

$$
\int_{0}^{\infty} e^{-2 \gamma t} t^{-2 j}|| u\left\|_{L^{2}\left(R_{+}^{n}\right)}^{2} d t \leqq C \int_{0}^{\infty} e^{-2 \gamma t} t^{-2(j-1)}\right\| u_{t} \|_{L^{2}\left(R_{+}^{n}\right)}^{2} d t
$$

we have

$$
\begin{aligned}
& \Psi_{m}(u) \leqq C\left\{\gamma^{-1} \delta \sum_{|\alpha|+j \leq m-1}\left(\left\|t^{-1} D_{t y}^{\alpha} D_{x}^{j} u\right\|_{\mathscr{H}_{-\gamma}}^{2}+\gamma^{-2}\left\|t^{-2} D_{t y}^{\alpha} D_{x}^{j} u\right\|_{\mathscr{H}_{-\gamma}}^{2}\right)\right. \\
& \quad+(1+\gamma \mu)\|u\|_{\mathscr{H}_{m-1,-\gamma}}+\gamma^{-3} \delta\left\|t^{-1} \tilde{P}_{m \varepsilon} u\right\|_{\mathscr{H}_{-\gamma}}^{2} \\
& \left.\quad+(1+\gamma \mu)\left\|\Lambda^{-1} \tilde{P}_{m \varepsilon} u\right\|_{\mathscr{H}_{-\gamma}}^{2}\right\} .
\end{aligned}
$$

Now we introduce some notations for convenience. For any integer $k \geqq 0$, we denote

$$
\begin{aligned}
& \Psi_{k}^{(1)}(u)=\sum_{|\alpha|+i \leq m-1} \sum_{|\beta|=k}\left\|D_{t y}^{\alpha+\beta} D_{x}^{i} u\right\|_{\mathscr{H}_{-\gamma}}^{2}, \\
& \Psi_{k}^{(2)}(u)=\sum_{|\alpha|+i \leq m-1} \sum_{|\beta|=k}\left\|t^{-1} D_{t y}^{\alpha+\beta} D_{x}^{i} u\right\|_{\mathscr{H}}^{2}-\gamma^{9} \\
& \Psi_{k}^{(3)}(u)=\left.\sum_{|\alpha|+i \leq m-1} \sum_{|\beta|=k} \gamma^{-2}|| t^{-2} D_{t y}^{\alpha+\beta} D_{x}^{i} u\right|_{\mathscr{H}_{-}} ^{2}
\end{aligned}
$$

Then we observe that 


$$
\begin{aligned}
& \Psi_{m}(u) \leqq C\left\{\sum_{i=2}^{3} \delta \gamma^{-1} \Psi_{0}^{(i)}(u)+\Psi_{0}^{(1)}(u)+\gamma \mu \Psi_{0}^{(1)}(u)\right. \\
&\left.+\gamma^{-1} \sum_{j=0}^{1} \delta^{j}\left\|(\gamma t)^{-j} \tilde{P}_{m \varepsilon} u\right\|_{\mathscr{C}_{-\gamma}}^{2}\right\} .
\end{aligned}
$$

If we take $t^{m_{j}+1} P_{j 0, \delta} \circ \phi_{0} t^{-m_{j}-1} u+\left(P_{j 0, r}+P_{j 0, \mu}\right) \circ \phi_{0} u$ instead of $u$ in the statement of Lemma 4.6, using Remark 5 and Lemma $4.4^{\prime}$ we have

a) ${ }_{j} \quad c_{\mu} \gamma\left\|P_{j 0} \phi_{0} u\right\|_{\mathscr{H}_{m_{j}-1,-r}}^{2}+\sum_{k=m_{j}^{+}}^{m_{j}-1}\left\langle\left\langle P_{j_{k}} \phi_{0} u\right\rangle_{\mathscr{H}_{m_{j}-1-k_{0}-r}}^{2}\right.$

$$
\begin{aligned}
& \leqq C \mu^{2} \sum_{k=0}^{m_{j}^{+}-1}\left\langle P_{j k} \phi_{0} u\right\rangle_{\mathscr{H}_{m_{j}-1-k,-r}}^{2}+C_{\mu}\left\{\gamma^{-1} \sum_{j=0}^{1} \delta^{j}\left\|(\gamma t)^{-j} \tilde{P}_{m \varepsilon} u\right\|_{\mathscr{H}_{-}}^{2}\right. \\
& \left.+\gamma \mu \Psi_{0}^{(1)}\left(\phi_{0} u\right)+\Psi_{0}^{(1)}(u)+\gamma^{-1} \delta \sum_{i=2}^{3} \Psi_{0}^{(i)}(u)\right\}
\end{aligned}
$$

for arbitrary $0<\mu<1$ and $j=1, \ldots, h$. If we take

$$
t^{m_{0}^{ \pm}+1} Q_{0 \diamond}^{ \pm} \circ \phi_{0} t^{-m_{0}^{ \pm}-1} u+\left(Q_{0 r}^{ \pm}+Q_{0 \mu}^{ \pm}\right) \circ \phi_{0} u
$$

instead of $u$ in the statement of Lemma 4.7, we have similarly

b) $\gamma\left\|Q_{0}^{+} \phi_{0} u\right\|_{\mathscr{H}_{m_{0}^{+}-1,-\gamma}}^{2} \leqq C\left\{\sum_{k=0}^{m_{0}^{+}-1}\left\langle\left\langle Q_{k}^{+} \phi_{0} u\right\rangle_{\mathscr{H}_{m_{0}^{+}-1-k,-\gamma}}^{2}\right.\right.$

$$
\begin{aligned}
& +\gamma^{-1} \sum_{j=0}^{1} \delta^{j}\left\|(\gamma t)^{-j} \tilde{P}_{m \varepsilon} u\right\|_{\mathscr{H}_{-\gamma}}^{2}+\gamma \mu \Psi_{0}^{(1)}\left(\phi_{0} u\right)+\Psi_{0}^{(1)}(u) \\
& \left.+\gamma^{-1} \sum_{i=2}^{3} \delta \Psi_{0}^{(i)}(u)\right\} .
\end{aligned}
$$

c) $\gamma\left\|Q_{0}^{-} \phi_{0} u\right\|_{\mathscr{H}_{m_{0}^{-}-1,-\gamma}^{2}}^{2}+\sum_{k=0}^{m_{0}^{-}-1}\left\langle Q_{\bar{k}}^{-} \phi_{0} u\right\rangle_{\mathscr{H}_{m_{0}^{-}-1-k,-r}}^{2}$

$$
\begin{aligned}
& \leqq C\left\{\gamma^{-1} \sum_{j=0}^{1} \delta^{j}\left\|(\gamma t)^{-j} \tilde{P}_{m \varepsilon} u\right\|_{\mathscr{H}_{-\gamma}}^{2}+\gamma \mu \Psi_{0}^{(1)}\left(\phi_{0} u\right)+\Psi_{0}^{(1)}(u)\right. \\
& \left.+\gamma^{-1} \delta \sum_{i=2}^{3} \Psi_{0}^{(i)}(u)\right\} .
\end{aligned}
$$

By Proposition 1 and combining a) ${ }_{j}$, b) and c) we have for sufficiently small $\mu$,

$$
\begin{aligned}
& \sum_{j=0}^{m-1}\left\langle D_{x}^{j} \phi_{0} u\right\rangle_{\mathscr{H}_{m-1-j,-\gamma}^{2}}^{2} \leqq C_{1}\left\{\gamma^{-1} \sum_{j=0}^{1} \delta^{j}\left\|(\gamma t)^{-j} \tilde{P}_{m \varepsilon} u\right\|_{\mathscr{H}_{-\gamma}}^{2}\right. \\
& \quad+\sum_{j=1}^{m_{+}}\left\langle\left\langle\tilde{B}_{j 0, \varepsilon} u\right\rangle_{\mathscr{H}_{m-1-r_{j},-\gamma}}^{2}+\gamma \mu \Psi_{0}^{(1)}\left(\phi_{0} u\right)+\Psi_{0}^{(1)}(u)\right\}+c_{\phi_{0}} \delta \gamma^{-1} \sum_{i=2}^{3} \Psi_{0}^{(i)}(u) .
\end{aligned}
$$

Since $P_{j k}\left(1 \leqq j \leqq h, 0 \leqq k \leqq m_{j}-1\right)$ and $Q_{k}^{ \pm}\left(0 \leqq k \leqq m_{0}^{ \pm}-1\right)$ form a basis of $L$, by adding the inequalies a) ${ }_{j}$, b) and c) and using the just 
obtained estimate we obtain

$$
\begin{aligned}
& \text { (4.31) } 2 \gamma \|\left.\phi_{0} u\right|_{\mathscr{H}_{m-1,-\gamma}} ^{2}+\sum_{j=0}^{m-1}\left\langle\left\langle D_{x}^{j} \phi_{0} u\right\rangle_{\mathscr{H}_{m-1-j,-\gamma}}^{2} \leqq C_{2}\left\{\gamma^{-1} \sum_{j=0}^{1} \delta^{j}||(\gamma t)^{-j} \tilde{P}_{m \varepsilon} \|_{\mathscr{H}_{-\gamma}}^{2}\right.\right. \\
& +\sum_{j=1}^{m}\left\langle\left\langle\tilde{B}_{j 0, \varepsilon} u\right\rangle_{\mathscr{H}_{m-1-r_{j},-r}}^{2}+\gamma \mu \Psi_{0}^{(1)}\left(\phi_{0} u\right)+\Psi_{0}^{(1)}(u)\right\} \\
& +c_{\phi_{0}}^{\prime} \delta \gamma^{-1} \sum_{i=2}^{3} \Psi_{0}^{(i)}(u) \text { 。 }
\end{aligned}
$$

By using the following inequality

$$
\begin{aligned}
& C_{2}\left(\gamma^{-1} \sum_{j=0}^{1} \delta^{j}\left\|(\gamma t)^{-j}\left(\tilde{P}_{\varepsilon}-\tilde{P}_{m \varepsilon}\right) u\right\|_{\mathscr{H}_{-\gamma}}^{2}+\sum_{j=1}^{m_{+}}\left\langle\left(\tilde{B}_{j \varepsilon}-\tilde{B}_{j 0, \varepsilon}\right) u\right\rangle_{\mathscr{H}_{m-1-\gamma_{j},-\gamma}}^{2}\right) \\
& \quad \leqq C_{\phi_{0}} \gamma^{-1} \Psi_{0}^{(2)}(u)+C_{\phi_{0}}^{\prime} \gamma^{-1} \delta \Psi_{0}^{(3)}(u)
\end{aligned}
$$

and (4.31) we have

$$
\begin{aligned}
& \left(2-C_{2} \mu\right) \gamma\left\|\phi_{0} u\right\|_{\mathscr{H}_{m-1,-\gamma}}^{2}+\sum_{j=1}^{m-1}\left\langle\left\langle D_{x}^{j} \phi_{0} u\right\rangle_{\mathscr{H}_{m-1-j_{,}-r}^{2}}\right. \\
& \quad \leqq C_{2}\left\{\gamma^{-1} \sum_{j=0}^{1} \delta^{j}\left\|(\gamma t)^{-j} \tilde{P}_{\varepsilon} u\right\|_{\mathscr{H}_{-\gamma}}^{2}+\sum_{j=1}^{m_{+}}\left\langle\tilde{B}_{j \varepsilon} u\right\rangle_{\mathscr{H}_{m-1-r_{j^{\prime}}-\gamma}}+\Psi_{0}^{(1)}(u)\right\} \\
& \quad+C_{\phi_{0}} \gamma^{-1} \Psi_{0}^{(2)}(u)+c_{\phi_{0}}^{\prime \prime} \delta \gamma^{-1} \sum_{i=2}^{3} \Psi_{0}^{(i)}(u) .
\end{aligned}
$$

Put $\mathscr{K}_{-}=\left\{(\tau, \eta) \in \boldsymbol{C}^{1} \times\left.\mathbb{R}^{n-1}|\operatorname{Im} \tau \leqq 0,| \tau\right|^{2}+|\eta|^{2}=1\right\}$ 。 We can choose a finite open covering $\left\{U\left(X_{k}\right): X_{k}=\left(t_{k}, x_{k}, y_{k} ; \tau_{k}, \eta_{k}\right) \in \tilde{K} \times \mathscr{K}_{-}\right\}_{k=1}^{J}$ of $\tilde{K} \times \mathscr{K}$ - where $\tilde{K}=\left\{(t, x, y) \in \mathbb{R}^{n+1}||(t, x, y) \mid \leqq T_{2}\right\}$ and $T_{2}=\max \left\{T_{0}\right.$, $\left.T_{0}^{k}\right\}$. Then we consider a partition of unity $\left\{\phi_{k}\right\}_{k=1}^{J}$ crresponding to open covering and we take every $U\left(X_{k}\right)$ so small that for every $\phi_{k}$ (4.32) holds instead of $\phi_{0}$. Put $\alpha=\sum_{k=1}^{J} \phi_{k}$. For $|\beta| \leqq s$ we have

$$
\begin{aligned}
& \left\|\left[\tilde{P}_{\varepsilon}, D_{t y}^{\beta}\right] u\right\|_{\mathscr{H}_{-r}}^{2} \leqq C\left\{\Psi_{s}^{(2)}(u)+\Psi_{s}^{(1)}(u)\right\}, \\
& \quad\left\langle\left[\tilde{B}_{j \varepsilon}, D_{t y}^{\beta}\right] u\right\rangle_{\mathscr{H}_{m-1-\gamma_{j},-r}}^{2} \leqq C \gamma^{-1}\left\{\Psi_{s}^{(2)}(u)+\Psi_{s}^{(1)}(u)\right\} 。
\end{aligned}
$$

Connecting the above inequalities with (4.32) implies that for every $\phi_{k}$ there exist constants $C(k), C_{\phi_{k}}$ and $c_{\phi_{k}}$ such that

$$
\begin{aligned}
(4.33)_{k} \sum_{|\alpha| \leqq s} & \left\{\sum_{j=0}^{m-1}\left\langle\left\langle D_{x}^{j} D_{t y}^{\alpha} \phi_{k} u\right\rangle_{\mathscr{H}_{m-1-j_{0}-\gamma}^{2}}+(2-C(k) \mu) \gamma \|\left. D_{t y}^{\alpha} \phi_{k} u_{\|^{\prime}}\right|_{\mathscr{H}_{m-1,-\gamma}} ^{2}\right\}\right. \\
\leqq & C(k) \sum_{|\beta| \leqq s}\left\{\gamma^{-1} \sum_{j=0}^{1}\left\|(\gamma t)^{-j} D_{t y}^{\beta} \tilde{P}_{\varepsilon} u\right\|_{\mathscr{H}_{-\gamma}}^{2}+\sum_{j=1}^{m_{+}}\left\langle\left\langle D_{t y}^{\beta} \tilde{B}_{j \varepsilon} u\right\rangle_{\mathscr{H}_{m-1-r_{j^{\prime}}-r}^{2}}\right.\right. \\
& \left.+\Psi_{s}^{(1)}(u)\right\}+\gamma^{-1} C_{\phi_{k}} \Psi_{s}^{(2)}(u)+c_{\phi_{k}} \delta \gamma^{-1} \sum_{i=2}^{3} \Psi_{s}^{(i)}(u) .
\end{aligned}
$$

Adding $(4.33)_{k}$ for $1 \leqq k \leqq J$ there exist constants $C_{\alpha}$ and $c_{\alpha}$ such that 


$$
\begin{aligned}
& \text { for } C(\alpha)=\max _{1 \leqq k \leqq J} C(k) \\
& \text { (4.34) } \quad(2-G(\alpha) \mu) \gamma_{s}^{(1)}(\alpha u) \\
& \leqq C_{3} \sum_{|\beta| \leqq s}\left\{\gamma^{-1} \sum_{j=0}^{1}\left\|(\gamma t)^{-j} D_{t y}^{\beta} \tilde{P}_{\varepsilon} u\right\|_{\mathscr{H}_{-\gamma}}^{2}+\sum_{j=1}^{m_{+}}\left\langle D_{t y}^{\beta} \tilde{B}_{j \varepsilon} u\right\rangle_{\mathscr{H}_{m-1-r_{j^{\prime}}-\gamma}}\right. \\
& +\Psi_{s}^{(1)}(u)+\gamma^{-1} C_{\alpha} \Psi_{s}^{(2)}(u)+c_{\alpha} \delta \gamma^{-1} \sum_{i=2}^{3} \Psi_{s}^{(i)}(u) 。
\end{aligned}
$$

Now we take $\mu$ so small that $2-C(\alpha) \mu>0$.

Recalling that the coefficients of $\left\{\tilde{P}_{\varepsilon}, \tilde{B}_{j \varepsilon}\right\}$ are constant in $R^{n+1} \backslash \tilde{K}$, following to [9] we have

$$
\begin{aligned}
& 2 \gamma \Psi_{s}^{(1)}((1-\alpha) u) \\
& \quad \leqq C_{4} \sum_{|\beta| \leqq s}\left\{\gamma^{-1}\left\|D_{t y}^{\beta} \tilde{P}_{\varepsilon} u\right\|_{\mathscr{H}_{-\gamma}}^{2}+\sum_{j=1}^{m_{+}}\left\langle D_{t y}^{\beta} \tilde{B}_{j \varepsilon} u\right\rangle_{\mathscr{H}_{m-1-r_{j},-\gamma}}^{2}\right\} \\
& \quad+C_{5} \Psi_{s}^{(1)}(u) .
\end{aligned}
$$

Then, by (4.34) and (4.35) we have

$$
\begin{aligned}
& (2-\nu) \gamma \Psi_{s}^{(1)}(u) \leqq C_{6} \sum_{|\beta| \leqq s}\left\{\gamma^{-1} \sum_{j=0}^{1}\left\|_{i}(\gamma t)^{-j} D_{t y}^{\beta} \tilde{P}_{\varepsilon} u\right\|_{\mathscr{H}}^{2}-\gamma\right. \\
& \quad+\sum_{j=1}^{m_{+}}\left\langle\left\langle D_{t y}^{\beta} \tilde{B}_{j \varepsilon}\right\rangle_{\mathscr{H}^{2}-1-r_{j},-\gamma}^{2}\right\}+\gamma^{-1} C_{\alpha} \Psi_{s}^{(2)}(u)+c_{\alpha} \delta \gamma^{-1} \sum_{j=2}^{3} \Psi_{s}^{(i)}(u) .
\end{aligned}
$$

where $\nu=C(\alpha) \mu+\left(C_{5}+C_{3}\right) \gamma^{-1}$. Now we take $\gamma_{1}$ so large that for any $\gamma \geqq \gamma_{1}$ it holds $2-\nu>0$. Then we note that we can take $C_{6}$ independent of such $\gamma_{0}$ Let $M(s) \geqq 4\left(C_{\alpha}+2 \mathrm{c}_{\alpha} \delta\right)(2-\nu)^{-1}+3$.

Since it was shown in [9] that the solution $u$ of (1.4) is independent of $\gamma$, by integration by parts, it holds

$$
\begin{aligned}
\gamma \int_{0}^{\infty} e^{-2 \gamma t} \sum_{k=0}^{m-1}\left\|D_{t}^{k} u\right\|_{H_{m-1-k}\left(\boldsymbol{R}_{+}^{n}\right)}^{2} d t \\
=-1 / 4(\partial / \partial \gamma) \int_{0}^{\infty} \sum_{k=0}^{m-1} e^{-2 \gamma t}|| t^{-1} D_{t}^{k} u \|_{H_{m-1-k}\left(\boldsymbol{R}_{+}^{n}\right)}^{2} d t \\
\quad-1 / 2(\partial / \partial \gamma) \int_{0}^{\infty} t^{-1} e^{-2 \gamma t} \gamma \sum_{k=0}^{m-1}\left\|D_{t}^{k} u\right\|_{H_{m-1-k}\left(\boldsymbol{R}_{+}^{n}\right)}^{2} d t
\end{aligned}
$$

Multiplying the both sides of $(4.36)$ by $2(2-\nu)^{-1} \gamma^{[M]+1}$, by the above equality we have

$$
\begin{aligned}
(4.37)- & 1 / 2(\partial / \partial \gamma)\left\{\gamma^{[M]+1} \Psi_{s}^{(2)}(u)\right\} \\
& -(\partial / \partial \gamma)\left\{\gamma^{[M]+2} \int_{0}^{\infty} t^{-1} e^{-2 \gamma t} \sum_{|\alpha| \leqq s} \sum_{k=0}^{m-1}|| D_{t}^{k} D_{t y}^{\alpha} u \|_{H_{m-1-k}\left(R_{+}^{n}\right)}^{2} d t\right\} \\
& +\left\{([M]+1) / 2-2\left(C_{\alpha}+c_{\alpha} \delta\right)(2-\nu)^{-1}\right\} \gamma^{[M] \Psi_{s}^{(2)}(u)} \\
& \leqq C_{6} \sum_{|\beta| \leqq s} \gamma^{[M]}\left\{\left\|_{i}^{\prime} D_{t y}^{\beta} \tilde{P}_{\varepsilon} u\right\|_{\mathscr{H}_{-\gamma}}^{2}+\sum_{j=1}^{m_{+}} \gamma\left\langle\left\langle D_{t y}^{\beta} \tilde{B}_{j \varepsilon} u\right\rangle_{\mathscr{H}_{m-1-r_{j}}-\gamma}^{2}\right\}\right.
\end{aligned}
$$




$$
+2 c_{\alpha} \delta(2-\nu)^{-1} \gamma^{[M]} \Psi_{s}^{(3)}(u)
$$

We consider

$$
\begin{aligned}
& \int_{\gamma_{1}}^{\infty} \gamma^{[M]} \Psi_{s}^{(3)}(u) d \gamma \\
& \quad=\int_{\gamma_{1}}^{\infty} \int_{0}^{\infty} \gamma^{[M]-2} e^{-2 \gamma t} t^{-2} \sum_{|\alpha|+i \leqq m-1} \sum_{|\beta|=s}\left\|t^{-1} D_{t y}^{\alpha+\beta} D_{x}^{i} u\right\|_{L^{2}\left(R \times R_{+}^{n}\right)}^{2} d t d \gamma
\end{aligned}
$$

by Fubini's theorem and integration by parts with respect to $\gamma$

$$
\leqq \frac{2^{2}}{[M]([M]-1)} \int_{r_{1}}^{\infty} \gamma^{[M]} \Psi_{s}^{(2)}(u) d \gamma \leqq \int_{r_{1}}^{\infty} \gamma^{[M]} \Psi_{s}^{(2)}(u) d \gamma_{\circ}
$$

Integrating the both sides of (4.37) over $\left(\gamma_{1}, \infty\right)$ the above inequality implies that we have by the dominated convergence theorem

$$
\begin{aligned}
& \int_{r_{1}}^{\infty} \int_{0}^{\infty} \gamma^{[M]} t^{-2} \sum_{|\alpha| \leqq s} \sum_{k=0}^{m-1}\left\|D_{t}^{k} D_{t y}^{\alpha} u\right\|_{H_{m-1-k}\left(R_{+}^{n}\right)}^{2} e^{-2 \gamma t} d t d \gamma \\
& \leqq C_{7}\left\{\sum_{|\alpha| \leqq s} \int_{r_{1}}^{\infty} \gamma^{[M]}\left\|D_{t y}^{\alpha} \tilde{P}_{\varepsilon} u\right\|_{\mathscr{H}_{-\gamma}}^{2} d \gamma\right. \\
& \quad+\sum_{j=1}^{m} \int_{\gamma_{1}}^{\infty} \gamma^{[M]+1}\left\langle\left\langle D_{t y}^{\alpha} \tilde{B}_{j \varepsilon} u\right\rangle_{\mathscr{H}_{m-1-r_{j},-\gamma}^{2}} d \gamma\right\} .
\end{aligned}
$$

By Fubini's theorem and integration by parts with respect to $\gamma$ we have

$$
\begin{aligned}
\int_{0}^{\infty} & \sum_{j=0}^{[M]} t^{-j-3} \sum_{|\alpha| \leqq s} \sum_{k=0}^{m-1}\left\|D_{t y}^{\alpha} D_{t}^{k} u\right\|_{H_{m-1-k}\left(R_{+}^{n}\right)}^{2} e^{-2 \gamma_{1} t} d t \\
& \leqq C \sum_{i=0}^{[M]+1}\left\{\sum_{|\alpha| \leqq s} \int_{0}^{\infty} t^{-i}\left\|D_{t y}^{\alpha} \tilde{P}_{\varepsilon} u\right\|_{L^{2}\left(R_{+}^{n}\right)}^{2} d t\right. \\
& \left.+\sum_{j=0}^{m_{+}} \sum_{k=0}^{m-1-r_{j}} \int_{0}^{\infty} t^{-1-i}\left\|D_{t y}^{\alpha+k e_{0}} \tilde{B}_{j \varepsilon} u\right\|_{H_{m-1-r_{j}-k}\left(R^{n-1}\right)}^{2} d t\right\} .
\end{aligned}
$$

Remark 6. If $\tilde{P}$ and $\tilde{B}_{j}$ are the same operators as treated in $[8 ; 9]$, then taking account of Remark 3 we may take $\kappa=1, \delta=0$ and $0<\mu<1$ all through the above arguments. Clearly $C_{\alpha}$ may be taken 0 . Therefore we conclude the loss of regularity do not occur in this case and so the energy estimate obtained in the above takes the form of extension of one in $[8 ; 9]$.

The proof of Theorem 1.1. Let us choose $\tilde{N}(s)$ so that $2 \tilde{N}(s)+1$ $\geqq[M]+2$. Then on account of (1.3) by using Lemma 14.1 in Ohya [6] we have 
(4. 40)

$$
\begin{aligned}
& \sum_{|\alpha| \leqq s}\left\|D_{t y}^{\alpha} u\right\|_{H_{m-1}\left(\left(0, T_{1}\right) \times R_{+}^{n}\right)}^{2} \\
& \leqq G \sum_{|\alpha| \leqq s+\tilde{N}+1}\left\{\left\|D_{t y}^{\alpha} f_{1}\right\|_{L^{2}\left(\left(0, T_{1}\right) \times R_{+}^{n}\right)}^{2}\right. \\
& +\sum_{j=1}^{m_{+}}\left\|D_{t y}^{\alpha} g_{j 1}\right\|_{H_{m-1-r_{j}}}^{2}\left(\left(0, T_{1}\right) \times R^{n-1}\right\} .
\end{aligned}
$$

By making use of (4.28) and (4.29) we have for $|\alpha|=s$ and $h=1,2 .$. (4. 41) $\left\|D_{t y}^{\alpha} u\right\|_{H_{m-1+h}\left(\left(0, T_{1}\right) \times R_{+}^{n}\right)}^{2} \leqq C\left\{\left\|D_{t y}^{\alpha} \tilde{P}_{\varepsilon} u\right\|_{H_{h-1}\left(\left(0, T_{1}\right) \times R_{+}^{n}\right)}^{2}\right.$

$$
\left.+\sum_{|\beta|=1}\left\|D_{t y}^{\alpha+\beta} u\right\|_{H_{m+h-2}\left(\left(0, T_{1}\right) \times R_{+}^{n}\right)}^{2}\right\} \text {. }
$$

By using (4.41) for $h=1,2, \ldots$ successively, we conclude that

(4. 42) $\quad\|u\|_{H_{m+s-1}\left(\left(0, T_{1}\right) \times R_{+}^{n}\right)}^{2} \leqq C\left\{\left\|\tilde{P}_{\varepsilon} u\right\|_{H_{s-1}\left(\left(0, T_{1}\right) \times R_{+}^{n}\right)}^{2}\right.$

$$
\left.+\sum_{|\alpha| \leqq s}\left\|D_{t y}^{\alpha} u\right\|_{H_{m-1}}^{2}\left(\left(0, T_{1}\right) \times R_{+}^{n^{-}}\right)\right\}
$$

holds.

Combining (4.40) with (4.42) we obtain Theorem 1. 1.

The proof of (0.7). Let us go back to $t$-variable in (1.1) again. From (4.39) it follows

$$
\begin{aligned}
& \int_{0}^{T} t^{-\kappa([M]+3)} \sum_{|\alpha| \leqq s} \sum_{k=0}^{m-1}\left\|\left(t^{-(\kappa-1)} D_{t}\right)^{k+\alpha_{0}} D_{y}^{\alpha^{\prime}} u\right\|_{H_{m-1-k}\left(R_{+}^{n}\right)}^{2} t^{\kappa-1} d t \\
& \quad \leqq C \sum_{|\alpha| \leqq s} \sum_{i=0}^{[M]+1}\left\{\int_{0}^{T} t^{-\kappa i}\left\|\left(t^{-(\kappa-1)} D_{t}\right)^{\alpha_{0}} D_{y}^{\alpha{ }^{\prime}} t^{-\kappa m} f_{0}\right\|_{L^{2}\left(R_{+}^{n}\right)}^{2} t^{\kappa-1} d t\right. \\
& \quad+\sum_{j=1}^{m_{+}} \sum_{k+|\nu| \leqq m-1-r_{j}} \int_{0}^{T} t^{-\kappa(i+1)}\left\|\left(t^{-(\kappa-1)} D_{t}\right)^{\alpha_{0}+k} D_{y}^{\alpha^{\prime}+\nu} t^{-\kappa r_{j}} g_{j 0}\right\|_{L^{2}\left(R^{n-1}\right)}^{2} t^{\kappa-1} d t .
\end{aligned}
$$

Now for $G=([M]+1) \kappa+2 s(\kappa-1)+2 m \kappa-(\kappa-1), \quad G_{j}=([M]+2) \kappa+$ $2\left(s+m-1-r_{j}\right)(\kappa-1)+2 r_{j} \kappa-(\kappa-1)$, let

$$
N=\left\{\begin{array}{cc}
{[G / 2]+1} & (G>0) \\
0 & (G \leqq 0)
\end{array} \text { and } N_{j}=\left\{\begin{array}{cc}
{\left[G_{j} / 2\right]+1} & \left(G_{j}>0\right) \\
0 & \left(G_{j} \leqq 0\right)
\end{array}\right.\right.
$$

In case $\kappa \geqq 1$ by the same procedure from (4.40) to (4.42) we obtain (0.7). When $0<\kappa<1$, by taking $M$ so large that

$$
\kappa([M]+2)+1 \geqq-2(\kappa-1)(m-1+s),
$$

in the same way we have the desired result. 


\section{Appendix}

In $\S 1 \sim \S 4$ we considered the flat mixed problem for a degenerate hyperbolic equation. Here we shall consider a degenerate hyperbolic mixed problem without imposing the flatness at $t=0$ on data.

We define for a non-negative integer $l \leqq m$

$$
\begin{array}{r}
P=P\left(t, x, y ; D_{t}, D_{x}, D_{y}\right)=\sum_{s=0}^{l} t^{l-s} P_{m-s}\left(t, x, y ; D_{t}, D_{x}, D_{y}\right) \\
+\sum_{s=l+1}^{m} P_{m-s}\left(t, x, y ; D_{t}, D_{x}, D_{y}\right), \\
P_{s}=\sum_{i+j+|\nu|=s} a_{i j \nu}(t, x, y) t^{(\kappa-1)(j+|\nu|)} D_{t}^{i} D_{x}^{j} D_{y}^{\nu}(0 \leqq s \leqq m)
\end{array}
$$

with $a_{m 00} \neq 0, a_{0 m 0}=1$ and $B_{k}$ by $(0.3)$.

We assume that the coefficients of $\left\{P, B_{k}\right\}$ satisfy the following condition:

i) $\quad a_{i j \nu}, t^{(\kappa-1)(j+|\nu|)+l-s} a_{i j \nu}(i+j+|\nu|=m-s, 0 \leqq s \leqq l), t^{(\kappa-1)(j+|\nu|)} a_{i j \nu}$ $(i+j+|\nu| \leqq m-1-l), b_{i j \nu}$ and $t^{r(j+|\nu|)} b_{i j \nu}^{k}$ are in $\mathscr{B}^{\infty}\left((0, T) \times \mathbb{R}^{n}\right)$.

ii) The indicial equation associated with $P$ is defined by

$$
\mathscr{C}(\lambda, x, y)=\sum_{j=0}^{l} \lambda(\lambda-1) \ldots(\lambda-m+1+i) a_{j}(x, y)
$$

where $a_{j}(x, y)=a_{m-j, 0,0}(0, x, y)$. Then we assume that $\mathscr{C}(\lambda, x, y) \neq 0$ for any integer $\lambda \geqq m-l$ (see Tahara [11]).

Furthermore we assume $\left\{t^{m-l} P, B_{k}\right\}$ satisfy the assumption (A). Now we consider the mixed problem for $\left\{P, B_{k}\right\}$.

$$
\text { (M. P.) }\left\{\begin{array}{l}
P[u]=f(t, x, y) \text { in }(0, T) \times \mathbb{R}_{+}^{n} \\
B_{k}[u]=g_{k}(t, y), 1 \leqq k \leqq m_{+}, \text {on } x=0 \\
D_{t}^{j} u=h_{j}(x, y), 0 \leqq j \leqq m-1-l, \text { on } t=0 .
\end{array}\right.
$$

Theorem. For given $h_{j}(x, y) \in H_{\infty}\left(\mathbb{R}_{+}^{n}\right) \quad(j=0, \ldots, m-1-l), g_{k}(t, y)$ $\in H_{\infty}\left((0, T) \times \mathbb{R}^{n-1}\right)\left(k=1, \ldots, m_{+}\right)$and $f \in H_{\infty}\left((0, T) \times \mathbb{R}_{+}^{n}\right)$ there exists a unique solution $u \in H_{\infty}\left((0, T) \times \mathbb{R}_{+}^{n}\right)$ of the problem (M. P.), provided the data satisfy the compatibility condition of infinite order.

Proof. Let $u(t, x, y)$ be a smooth solution of the Cauchy problem

$$
\left\{\begin{array}{l}
P[u]=f \text { in }(0, T) \times \mathbb{R}_{+}^{n} \\
D_{t}^{j} u(0, x, y)=h_{j} \text { for } j=0, \ldots, m-1-l .
\end{array}\right.
$$


By Taylor's formula in $t$ we can express $u(t, x, y)$ in the form

$$
u(t, x, y)=\sum_{i=0}^{s-1}(1 / i !) t^{i} u_{i}(x, y)+t^{s} u_{s}(t, x, y)
$$

for $s \in \boldsymbol{N}$. Therefore under the assumptions i) and ii) we can uniquely determine the Taylor coefficients $\left\{u_{i}(x, y)\right\}_{i=0}^{s-1}$ inductively. Then, taking account of compatibility condition we can find $w \in C_{0}^{\infty}\left(\overline{\boldsymbol{R}_{+} \times \boldsymbol{R}_{+}^{n}}\right)$ so that $\left.D_{t}^{j}(f-P[w])\right|_{t=0}=0$ and $\left.D_{t}^{j}\left(g_{k}-B_{k}[w]\right)\right|_{t=0}=0$ for $j \geqq 0$ and $k=1, \ldots, m_{+}$. Hence (M. P.) is reduced to the following flat mixed problem

$$
\left\{\begin{array}{l}
t^{m-l} P[u]=t^{m-l}(f-P[w])=f_{0} \quad \text { in } \quad(0, T) \times \boldsymbol{R}_{+}^{n} \\
B_{k}[u]=g_{k}-B_{k}[w]=g_{k 0}, \quad 1 \leqq k \leqq m_{+}, \quad \text { on } \quad(0, T) \times \mathbb{R}^{n-1} \\
D_{t}^{j} u=0, \quad 0 \leqq j \leqq m-1, \quad \text { on } \quad t=0 .
\end{array}\right.
$$

Thus the proof can be accomplished by using the same technique as stated in $\S 1 \sim \S 4$.

\section{References}

[1] Calderón, A. P. and Zygmund, A., Singular integral operators and differential equations, Amer. J. Math., 79 (1957), 901-921.

[2] Kimura, K., A mixed problem for weakly hyperbolic equations of second order, Comm. in Partial Diff. Equations, 6 (1981), 1335-1361.

[3] Kumano-go, H., Pseudo-differential operators, MIT Press (1974).

[4] Nakamura, G. and Uryu, H., Parametrix of certain weakly hyperbolic operators, Comm. in Partial Diff. Equations, 5 (1980), 837-896.

[5] Mizohata, S., Theory of partial differential equations, Cambridge Univ. Press (1973).

[6] Ohya, Y., Le probléme de Cauchy á caracteristiques multiples, Ann. Scuola Norm. Sup. Pisa, 4 (1977) 757-805.

[ 7 ] Sakamoto, R., Iterated hyperbolic mixed problems, Publ. RIMS, kyoto Univ., 6 (1970), $1-44$.

[8] Mixed problems for hyperbolic equations I, II, J. Math. Kyoto Univ., 10 (1970).

[9] - Hyperbolic boundary value problems, Cambridge Univ. Press (1982).

[10] Mixed problems for degenerate hyperbolic equations, J. Math. Kyoto Univ., 23 (1983), 563-597.

[11] Tahara, H., In the Cauchy problem for Fuchsian hyperbolic Partial differential equations, J. Fac. Sci. Univ. Tokyo Sect. IA, 26 (1979). 